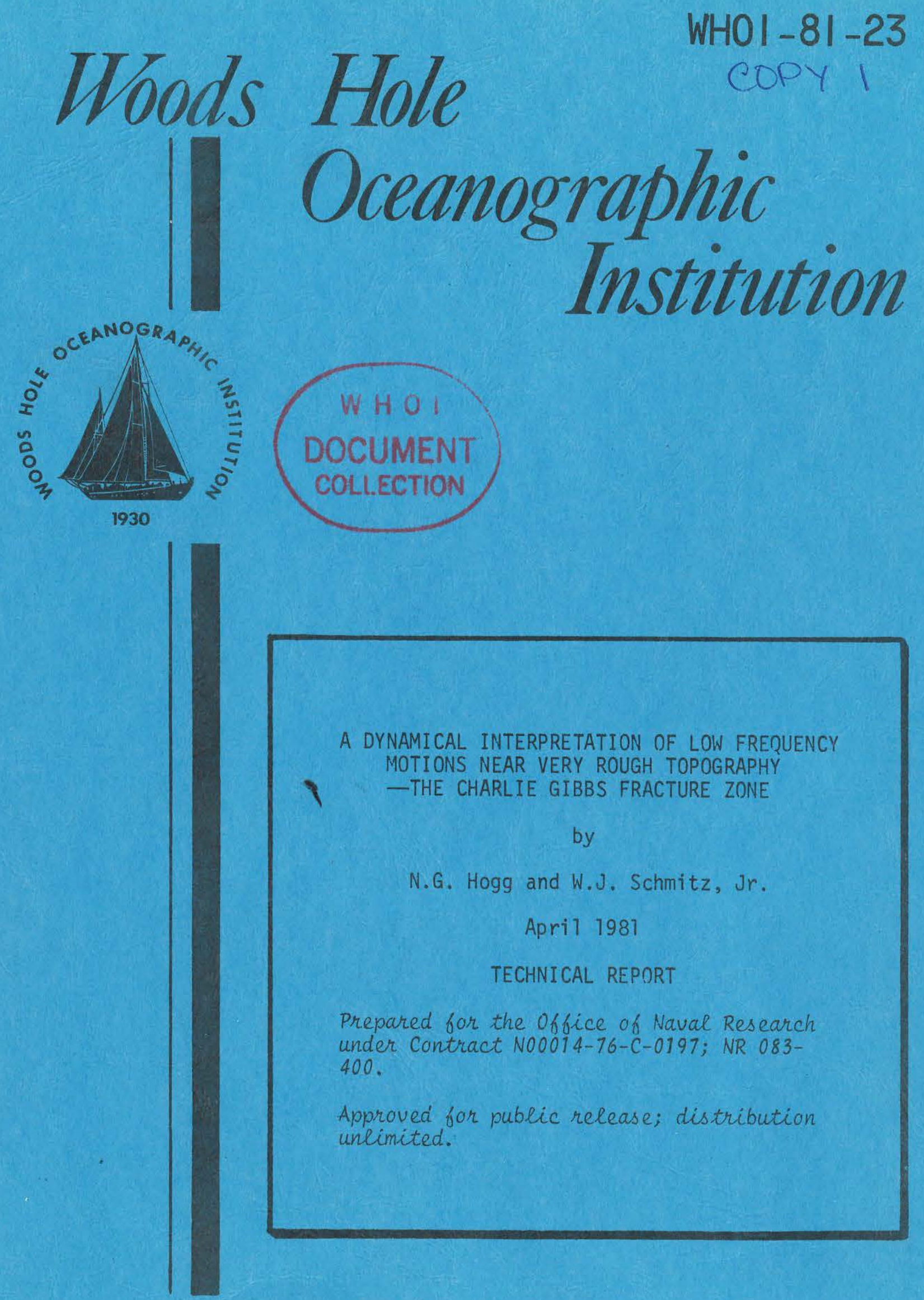

WOODS HOLE, MASSACHUSETTS 02543 


$$
\text { WHOI-81-23 }
$$

\title{
A DYNAMICAL INTERPRETATION OF LOW FREQUENCY MOTIONS NEAR VERY ROUGH TOPOGRAPHY \\ -THE CHARLIE GIBBS FRACTURE ZONE
}

by

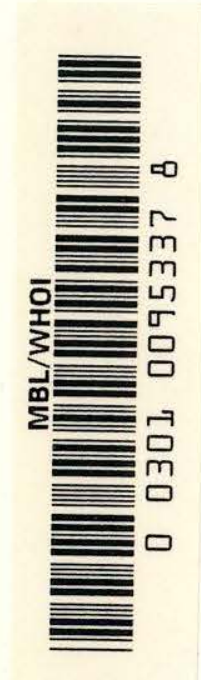

\author{
N.G. Hogg and W.J. Schmitz, Jr. \\ WOODS HOLE OCEANOGRAPHIC INSTITUTION \\ Woods Hole, Massachusetts 02543
}

Apri 11981

TECHNICAL REPORT

Prepared for the Office of Naval Research under Contract N00014-76-C-0197; NR 083-400.

Reproduction in whole or in part is permitted for any purpose of the United States Govermment. In citing this

report in bibliography, the reference given should be to: Joumal of Marine Research 38 (2): 215-248 (1980).

Approved for public release; distribution unlimited.

Approved for Distribution: Valentine Worthington, Chairman Department of Physical Oceanography 


\title{
A dynamical interpretation of low frequency motions near very rough topography-the Charlie Gibbs Fracture Zone
}

\author{
by $\mathbf{N}_{\bullet}$ G. Hogg ${ }^{1}$ and W. J. Schmitz, Jr. ${ }^{1}$
}

\begin{abstract}
As a sequel to Schmitz and Hogg (1978), nine-month moored observations of current and temperature from the Charlie Gibbs Fracture Zone are further described, and then interpreted in terms of low frequency quasigeostrophic motions. Large vertical and horizontal changes are observed in the variance of both mean and fluctuating components. It is demonstrated that these changes could be associated with the (complex) nature of the topography.

With regard to the mean flow, it is shown through an advective model that the topography is sufficiently steep to force this motion to closely follow isobaths. Time-dependent motions for periods from 2 to 96 days are described using the technique of empirical orthogonal functions. The most energetic mode is always bottom trapped, with flow oriented along isobaths at lower frequencies and approaching equipartition of along- and cross-isobath motions at higher frequencies. At the lowest frequencies a second mode which increases upward in energy is also judged significant, while for periods shorter than 3.6 days the second mode is again highly bottom trapped. We interpret these motions using linear wave theory. There is relatively close correspondence between theory and observation when the effects of both large- and small-scale topographic features are included in the model calculations. In addition to the usual topographic wave, the abrupt slope changes on the north wall allow for a baroclinic fringe mode with a near bottom node at low frequencies and small-scale bottom corrugations force highly bottom trapped waves above the smooth slope cut-off frequency.
\end{abstract}

\section{Introduction}

In a stratified ocean with gently sloping bottom, a class of transverse waves can exist (Rhines, 1970) with frequency proportional to the bottom slope and the cosine of the angle that the wavenumber vector makes with the isobaths. The maximum frequency $N \alpha$, the Brunt-Väisälä frequency times the bottom slope, is attained when the particle motions are perpendicular to the isobaths. Wavelengths shorter than the Rossby radius of deformation exponentially increase in amplitude toward the sea floor: they are "bottom trapped". Fluctuations with these general characteristics have been identified near Site D $(\sim 39 \mathrm{~N}, 70 \mathrm{~W})$ in the western North Atlantic (Thompson and Luyten, 1976; Thompson, 1977), a good place for comparison with idealized models because the isobaths are comparatively smooth and parallel. The

1. Woods Hole Oceanographic Institution, Woods Hole, Massachusetts, 02543, U.S.A. 


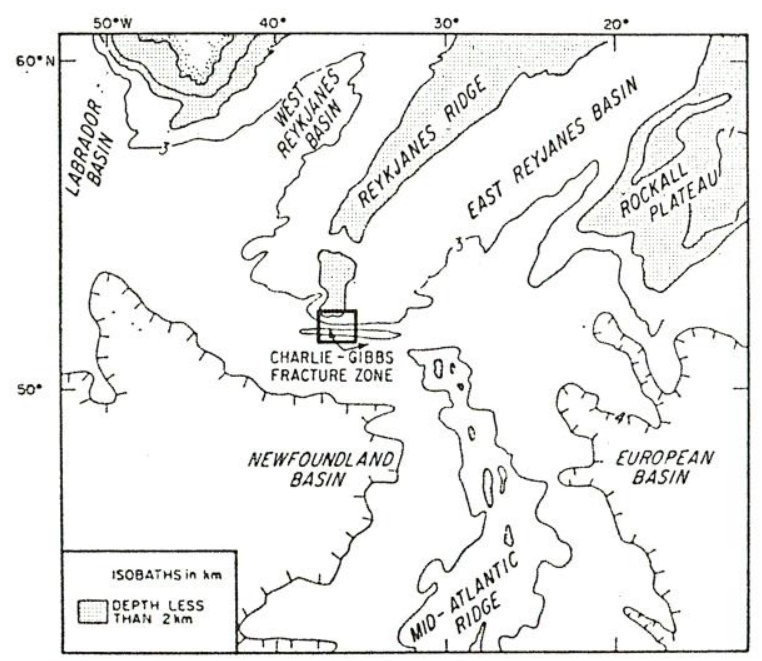

Figure 1. Bathymetry of a portion of the North Atlantic showing the relations of the Charlie Gibbs Fracture Zone to the Mid-Atlantic Ridge. Chart is courtesy of Sandy Shor (personal communication).

question arises, however, as to the applicability of these models to areas where isobaths are more contorted by, say, canyons or just the generally complex bathymetry of the Mid-Atlantic Ridge. The following contains an attempt in this context to dynamically interpret moored observations made in the Charlie Gibbs Fracture Zone (hereafter CGFZ) a deep east-west trough cutting through the Mid-Atlantic Ridge at 53W in the North Atlantic (Fig. 1).

Current meter moorings were deployed for nine months in September 1975 at the positions shown in Figure 2 and detailed in Table 1. Figure $2 \mathrm{a}$ is from Lonsdale and Shor (1979) and Shor, Lonsdale, Hollister, and Spencer (1980) while Figure 2b

Table 1. Mooring locations and depths, instrument depths, and data duration (from SH).

\begin{tabular}{|c|c|c|c|c|c|}
\hline Record \# & Location & $\begin{array}{l}\text { Depth } \\
\text { (m) }\end{array}$ & $\begin{array}{c}\text { Bottom } \\
\text { Depth (m) }\end{array}$ & Date Set & $\begin{array}{c}\text { Duration } \\
\text { (days) }\end{array}$ \\
\hline 5701 & $\begin{array}{l}52^{\circ} 42.7^{\prime} \mathrm{N} \\
33^{\circ} \\
59.2^{\prime} \mathrm{W}\end{array}$ & 4227 & 4311 & Sept. 26, 1975 & 269 \\
\hline 5711 & $\begin{array}{l}52^{\circ} 53.7^{\prime} \mathrm{N} \\
35^{\circ} 31.0^{\prime} \mathrm{W}\end{array}$ & 1007 & 2895 & Sept. 27, 1975 & 270 \\
\hline 5712 & & 2537 & & & 35 \\
\hline 5713 & & 2835 & & & 270 \\
\hline 5721 & $\begin{array}{l}52^{\circ} 46.1^{\prime} \mathrm{N} \\
35^{\circ} 30.0^{\prime} \mathrm{W}\end{array}$ & 988 & 3398 & Sept. 27, 1975 & 270 \\
\hline 5722 & & 2528 & & & 270 \\
\hline 5723 & & 3060 & & & 270 \\
\hline 5724 & & 3360 & & & 270 \\
\hline
\end{tabular}



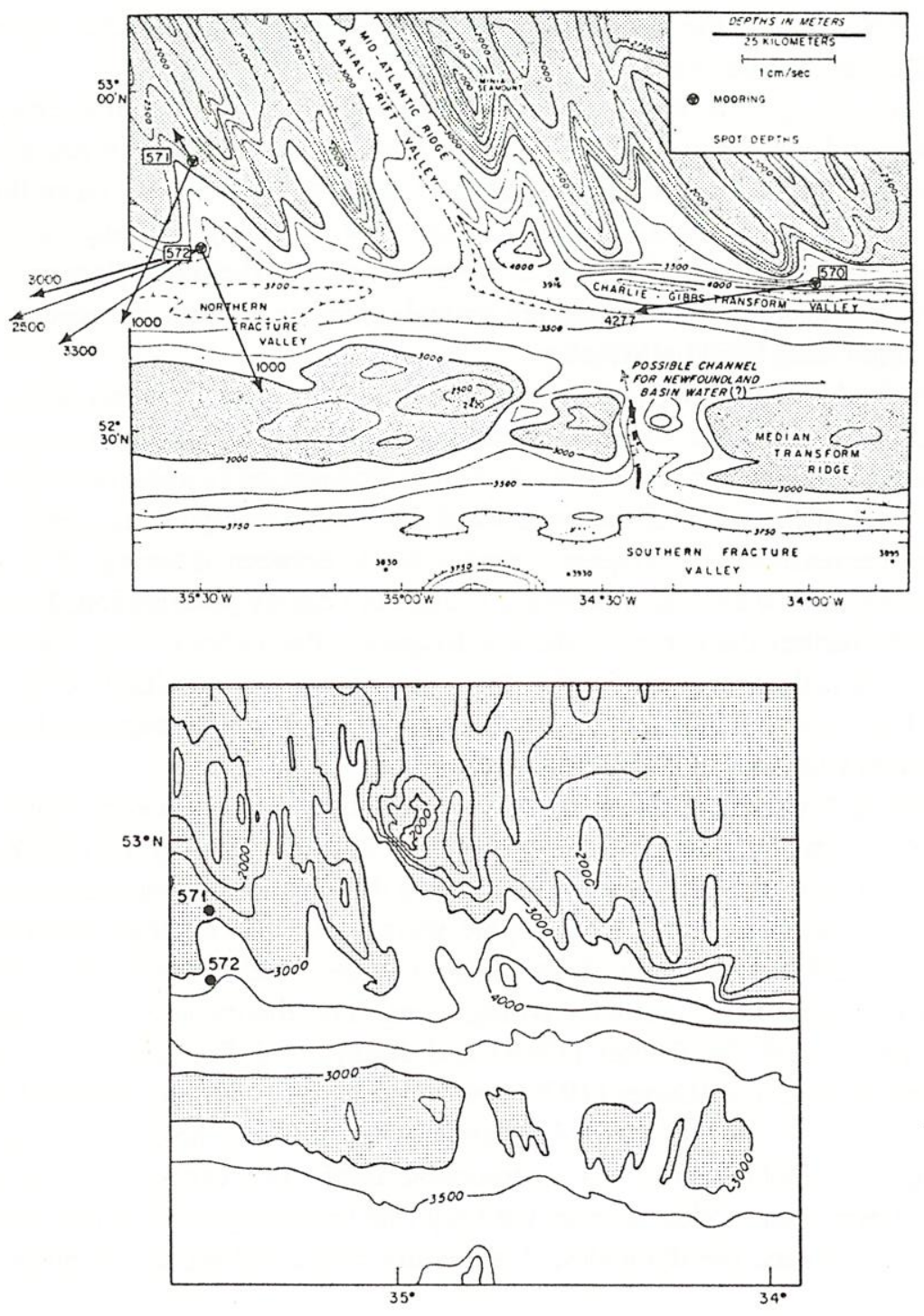

Figure 2. Two versions of the bathymetry within the box on Figure 1. (a) is interpolated from depth soundings and is adopted from Lonsdale and Shor (1979) and Shor et al. (1980). Arrows are mean flows measured at the indicated depths. (b) uses the same information as (a) with interpolation guided by side scanning sonar and the addition of one cruise track (DISCOVERY 84). It is traced from a chart prepared by Roger Searle (personal communication).

is redrawn from a chart prepared by Roger Searle at the Institute for Oceanographic Sciences, United Kingdom (personal communication) who used side scan sonar pictures as a guide for interpolation between the depth sounding tracks used in Figure 
2a. We shall explore the manner in which the geometrical character of the north wall influences the eddy field.

A principal objective of the mooring program was to explore properties of the mean flow and low frequency variability in this area with, results given in Schmitz and Hogg (1978) (hereafter SH) and Shor et al. (1980). The deep mean flow measured over 270 days, although always to the west, is highly spatially variable. We begin (Section 2) by briefly looking at the properties of the mean flow and demonstrate that the deep current vectors are strongly influenced, both in speed and direction, by small scale local bathymetry.

A principal result of $\mathrm{SH}$ is the discovery of an energetic, low frequency "eddy" field within CGFZ whose vertical structure changes with frequency from one which decreases in energy toward the bottom at low frequencies (period range of 48-500 days) to one which increases sharply toward the bottom at higher frequencies. There is a large decrease in low frequency energy levels between moorings 572 and 571 separated by just $10 \mathrm{~km}$ and a dramatic change in velocity polarization. In Section 3 we describe further the nature of the low frequency fluctuations using the technique of "empirical orthogonal functions"- the eigenfunctions of a suitable cross spectral matrix. The frequency domain from $1 / 96$ to $1 / 2$ c.p.d. can be separated into three bands within which the vertical structure changes only slowly.

In Section 4 we interpret these observations using low frequency wave theory. Section $4 \mathrm{a}$ formulates the problem and Section $4 \mathrm{~b}$ calculates, by two methods, the strength of nonlinearities. Above about .08 c.p.d. acceleration dominates advection. In Section $4 c$ we review quasigeostrophic wave theory on a broad, smooth slope. Sections $4 \mathrm{~d}$ and $4 \mathrm{e}$ deal with abrupt slope changes, while Section $4 \mathrm{f}$ tackles the problem of superposed sinusoidal corrugations. The theory is a modification and elaboration of work by Rhines (1969) and Rhines and Bretherton (1973) for a homogeneous case and Rhines (1970) and Suarez (1971) for the stratified problem and is discussed in terms of vertical dynamical modes. With the essential features of the topography included we find a reasonable qualitative explanation for the empirically determined modes, even in the very low frequency band where nonlinearities should influence the dynamics. A summary and conclusions are given in Section 5 .

The density stratification has an important influence on the dynamics both in determining the vertical modal structure and in determining the height of influence of small scale roughness. For future reference we reproduce in Figure 3 BruntVäisälä profiles $(N(z))$ from two stations near the mooring area. Below $1000 \mathrm{~m}$ depth $N(z)$ is roughly constant at 12 c./p.d.

\section{The mean flow}

Superimposed on the bathymetry in Figure $2 \mathrm{a}$ are the mean currents for the ninemonth mooring deployments. Substantial differences exist between moorings 571 


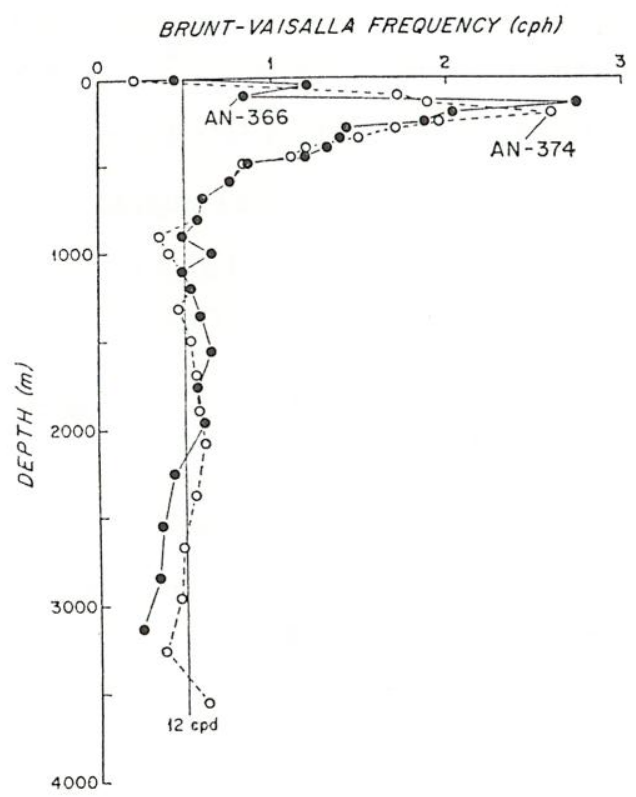

Figure 3. Two Brunt-Väisälä profiles from ANTON-DOHRN hydrographic stations taken near the mooring sites in April, 1964.

and 572 separated by just $10 \mathrm{~km}$ and there are also large vertical variations. We wish to explore the question of whether these observed differences in the deep current vectors could be a result of a small scale topographic constraint on a broader mean flow.

In the absence of dissipation the motion should conserve heat so that

$$
\vec{u}_{h} \cdot \nabla_{h} T+\frac{\partial \bar{T}}{\partial z} w=0
$$

$\vec{u}_{n}=(u, v)$ is the horizontal velocity, $w$ the vertical component, $T$ is temperature, and $\partial T / \partial z$ is the underlying vertical temperature gradient. Assuming that the vertical momentum balance is hydrostatic, the horizontal geostrophic, and that density perturbations are related to temperature perturbations through an expansion coefficient $\alpha_{T}$, then (1) with $N^{2}(z)=g \alpha_{T} T_{z}$ gives

$$
w=-\left(\frac{f}{N^{2}}\right)\left(u v_{z}-v u_{z}\right) .
$$

Another estimate for $w$ comes from the bottom boundary conditions:

$$
w=\vec{u}_{h} \cdot \nabla_{h} h @ z=-H+h(x, y) .
$$

On a single mooring (572, for instance) $\vec{u}_{h}$ is measured at two levels near the bottom so it is possible to derive a relation between $h_{x}$ and $h_{y}$ using (2) and (3). 
Hydrographic information from the area shows that density is little affected by salinity so that $\alpha_{T} \cong 1.7 \times 10^{-4} /{ }^{\circ} \mathrm{C}$. At $3300 \mathrm{~m} \vec{u}_{h}=(-3.2,-2.1) \mathrm{cm} / \mathrm{sec}$ and at $3000 \mathrm{~m} \vec{u}_{h}=(-3.8,-1.0) \mathrm{cm} / \mathrm{sec}$ (Table II in SH). From (2) and (3)

$$
3.2 h_{x}+2.1 h_{y}=.024 \mathrm{~cm} / \mathrm{sec} .
$$

If we ascribe an error of $\pm 1 \mathrm{~cm} / \mathrm{sec}$ to each velocity estimate, then the error in the R.H.S. of (4) is $\pm 36 \%$. The amplitude of the vertical velocity on the R.H.S. should be compared with $\left[\left(u h_{x}\right)^{2}+\left(v h_{y}\right)^{2}\right]^{1 / 2}$, the r.m.s. amplitude of the terms on the L.H.S. Using $\left|h_{\infty}\right| \cong\left|h_{y}\right| \cong 0.1$ as estimated from Figure 2 gives

$$
\frac{\vec{u}_{h} \cdot \nabla h}{\left[\left(u h_{x}\right)^{2}+\left(v h_{y}\right)^{2}\right]^{1 / 2}}=\frac{.024}{.38}=.063 .
$$

The topography is sufficiently steep so as to force motions to closely follow isobaths which, therefore, approximate streamlines. The differences in the near bottom mean vectors at 571 and 572 are, therefore, plausibly rationalized as due to different orientations and separations of the isobaths at the two locations.

\section{Time dependent motions}

The time dependent motions also exhibit large spatial variations which have been described in SH by identifying four frequency bands which contain motions with somewhat different characteristics: energy at very long periods (48 to 500 days) decreases toward the bottom while shorter periods are increasingly bottom intensified (see Figs. 2 and 4 from SH). This vertical structure and its changes with frequency will be compared to the dynamical vertical orthogonal modes resulting from linear quasigeostrophic wave theory. Making use of the vertical phase relationships between variables at different depths on a single mooring it is possible to calculate from the cross-spectral matrix "empirical orthogonal functions" which represent the vertical structure of the observed time-dependent motions in a statistically uncoupled sense. The degree to which one or more of these modes contain a significant portion of the variance are measures of the success of the technique. The reader is referred to Wallace and Dickinson (1972) for a formal discussion. In essence, given time series measurements at several vertical levels one computes the cross-spectral matrix containing all permutations between measured variables and levels. The empirical orthogonal functions are the eigenfunctions of the matrix and each function contains the fractional variance in the particular frequency band given by the ratio of its eigenvalue to the sum of all eigenvalues.

Cross-spectra were computed in a conventional manner: each time series was low-passed using a Gaussian filter with a 24-hour half power point (Schmitz, 1974) and then split into 10 overlapped pieces which were prewhitened, Fourier transformed, Hanned, piece averaged and then further frequency band-averaged over 


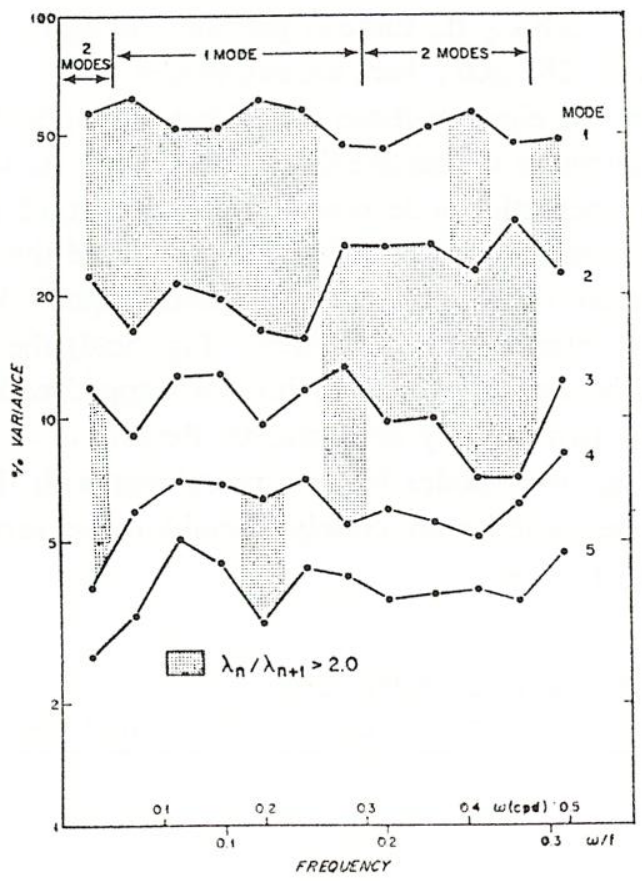

Figure 4. Percent variance versus frequency for the first five empirical orthogonal functions at mooring 572. Shaded regions indicate where a mode has twice the variance of the next higher mode.

two adjacent bands. Empirical modes were computed both with and without using temperature. The latter gave results more consistent with the kinetic energy distributions in $\mathrm{SH}$ and the dynamical analysis below. Coherence of temperature with velocity is generally low suggesting that the most energetic temperature modes are somewhat uncoupled from the principal velocity modes. We will interpret the most energetic velocity mode as a topographic wave: this being the barotropic mode it should be no surprise that temperature and horizontal velocity are uncoupled.

Figure 4 shows the percent variance contained in each empirical mode versus frequency. A homogeneous white noise process would result in indistinguishable variance contributions from each mode. Throughout the spectrum the first mode accounts for about 50 percent of the variance, while the second has 20 percent at low frequencies increasing to 30 percent at high frequencies. Wallace and Dickinson (1972) suggest that a $2: 1$ ratio of successive eigenvalues is an indication of significance although this is somewhat arbitrary. $\dagger$ These regions are shaded on Figure 4. The spectrum can be characterized by three different regimes; (i) "very low frequencies" the first band, there are 2-3 prominent modes, (ii) "intermediate frequen-

$\dagger$ At the $95 \%$ confidence level the Fisher $F$-test suggests that below a ratio of 2.12 successive eigenvalues are indistinguishable from homogeneous white noise with 20 degrees of freedom. 
cies," .05 c.p.d. $<\omega<.28$ c.p.d., there is just one distinguishable mode, and (iii) "high frequencies", $\omega>.28$ c.p.d., there are two modes.

In Figure 5 we show the eigenfunctions (logarithm of amplitude and phase) versus depth at selected frequencies characteristic of each band. At very low frequencies (Fig. 5a,b) the most energetic mode contains 58 percent of the variance and is oriented east-west with amplitude increasing slightly toward the bottom. The second mode contains 23 percent of the variance, split almost equally between components increasing upward. At intermediate frequencies (Fig. 5c,d) the first mode accounts for less variance, and becomes increasingly bottom trapped with more equipartition of energy between the two velocity components. Beyond $\omega=0.28$ c.p.d., at high frequencies (Figs. 5e-h), two modes become prominent with the first being highly bottom trapped and the second more closely resembling the vertical structure of the intermediate frequency modes.

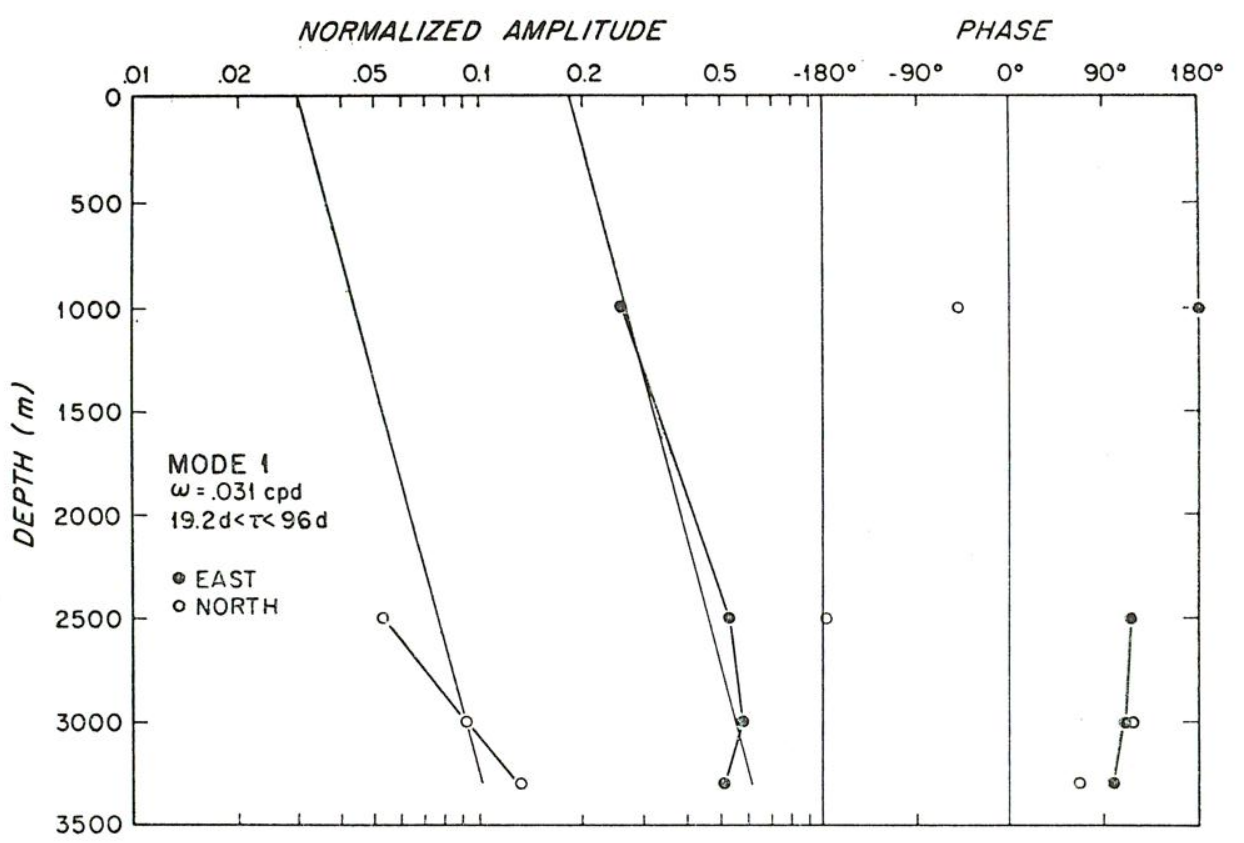

(a)

Figure 5. Empirical orthogonal functions, $\log _{10}$ (amplitude) and phase, at selected frequency estimates. For those modes which increase toward the bottom the straight lines are a least squares fit. For the amplitude portion only those points used in the fit are connected while only those estimates of phase which are significant at the $95 \%$ level are joined by lines. The short straight lines drawn on the high frequency plots $(\omega>.28$ c.p.d.) are the estimated decay rate of disturbances forced by $18 \mathrm{~km}$ corrugations. (a) Mode 1, $\omega=.031$ c.p.d. (b) Mode 2, $\omega=.031$ c.p.d. (c) Mode $1, \omega=.115$ c.p.d. (d) Mode 1, $\omega=.24$ c.p.d. (e) Mode $1, \omega=.323$ c.p.d. (f) Mode 2, $\omega=.323$ c.p.d. (g) Mode $1, \omega=.406$ c.p.d. (h) Mode 2, $\omega=.406$ c.p.d. 


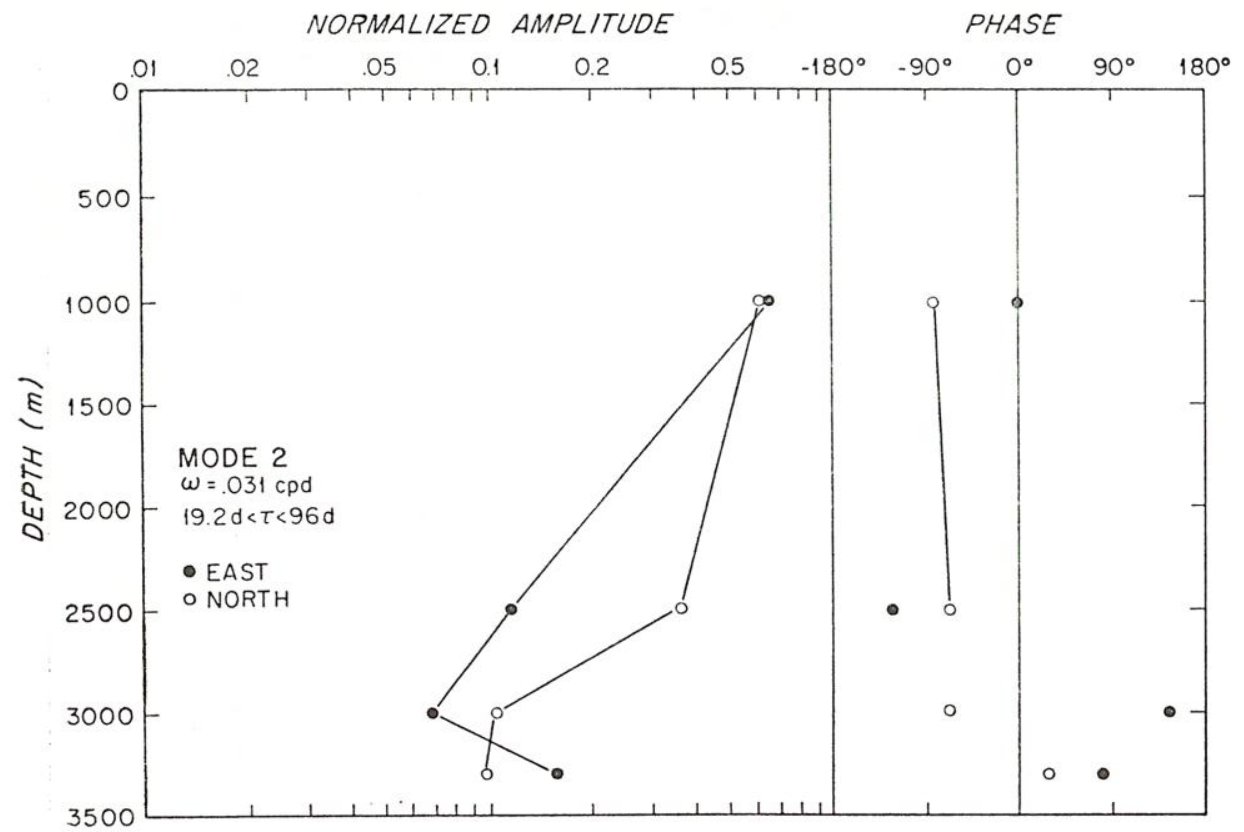

(b)

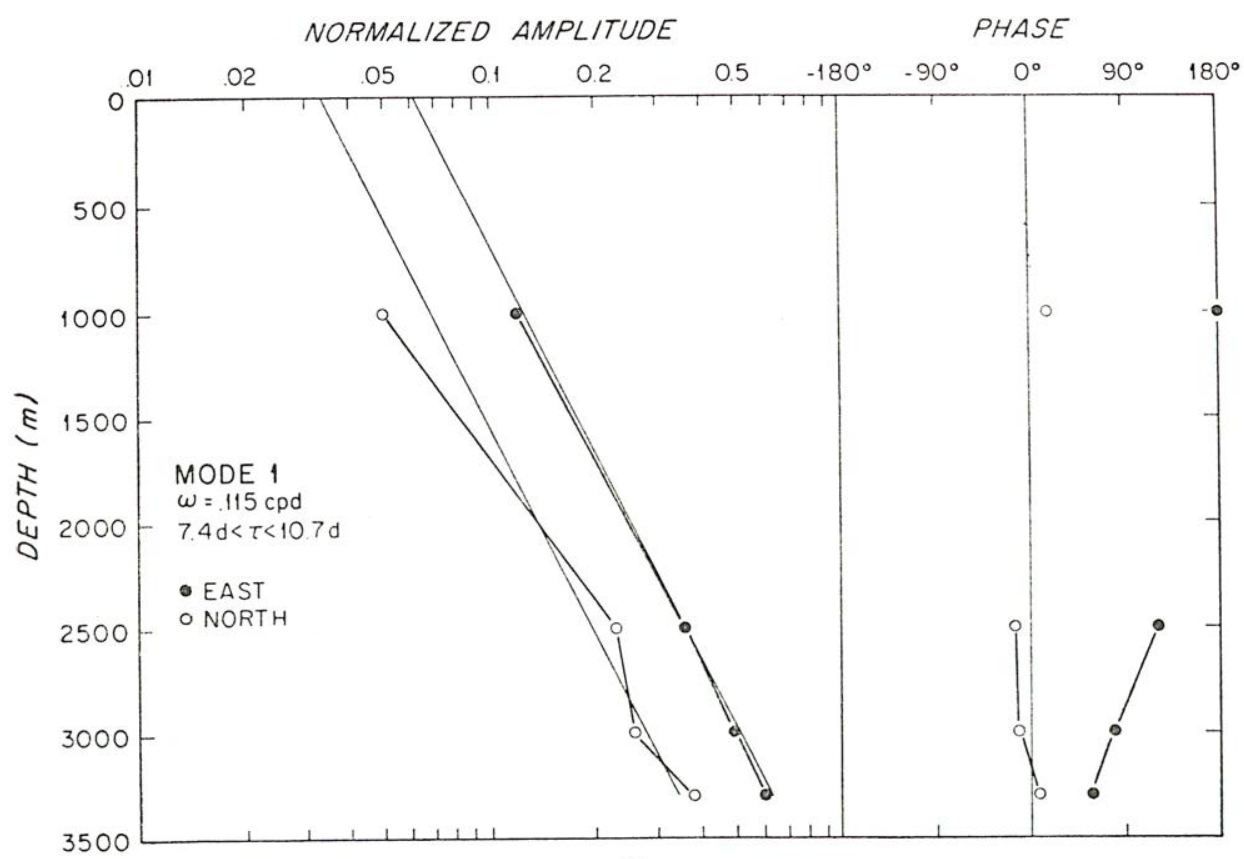

(c) 


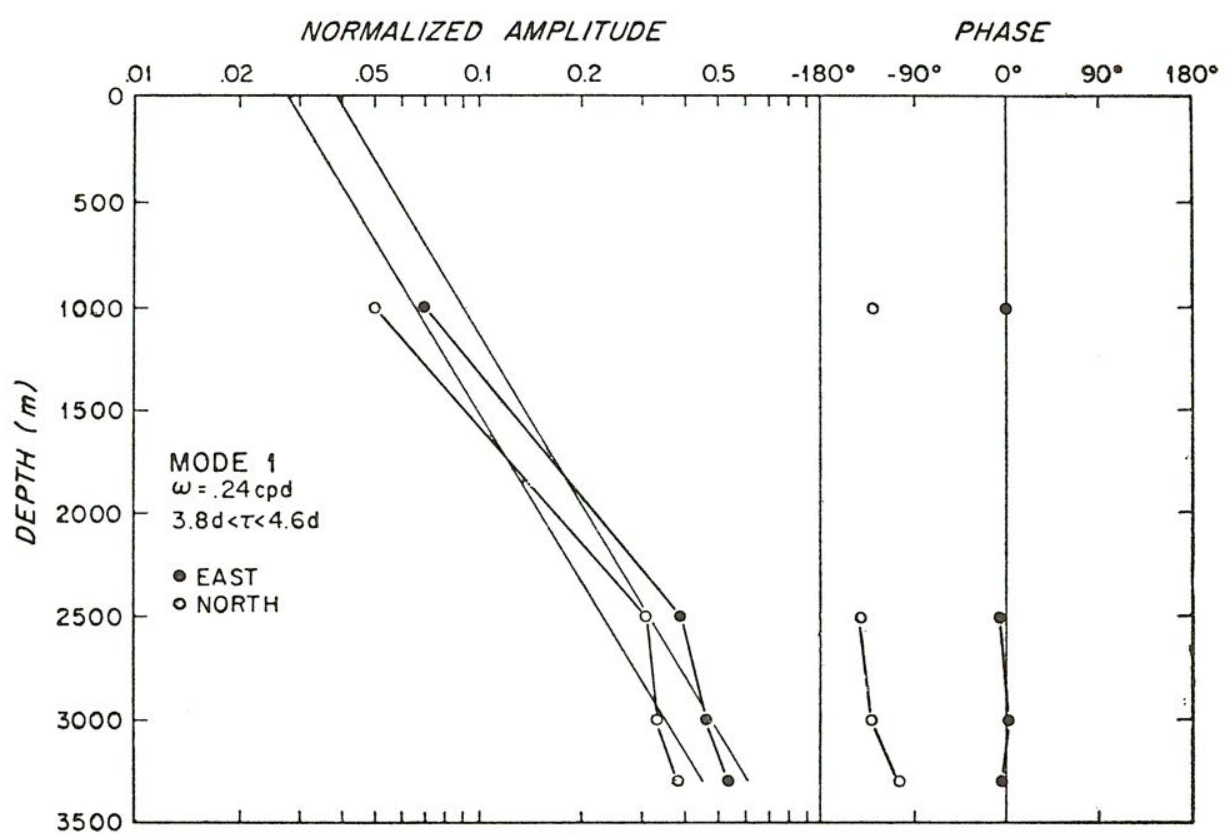

(d)

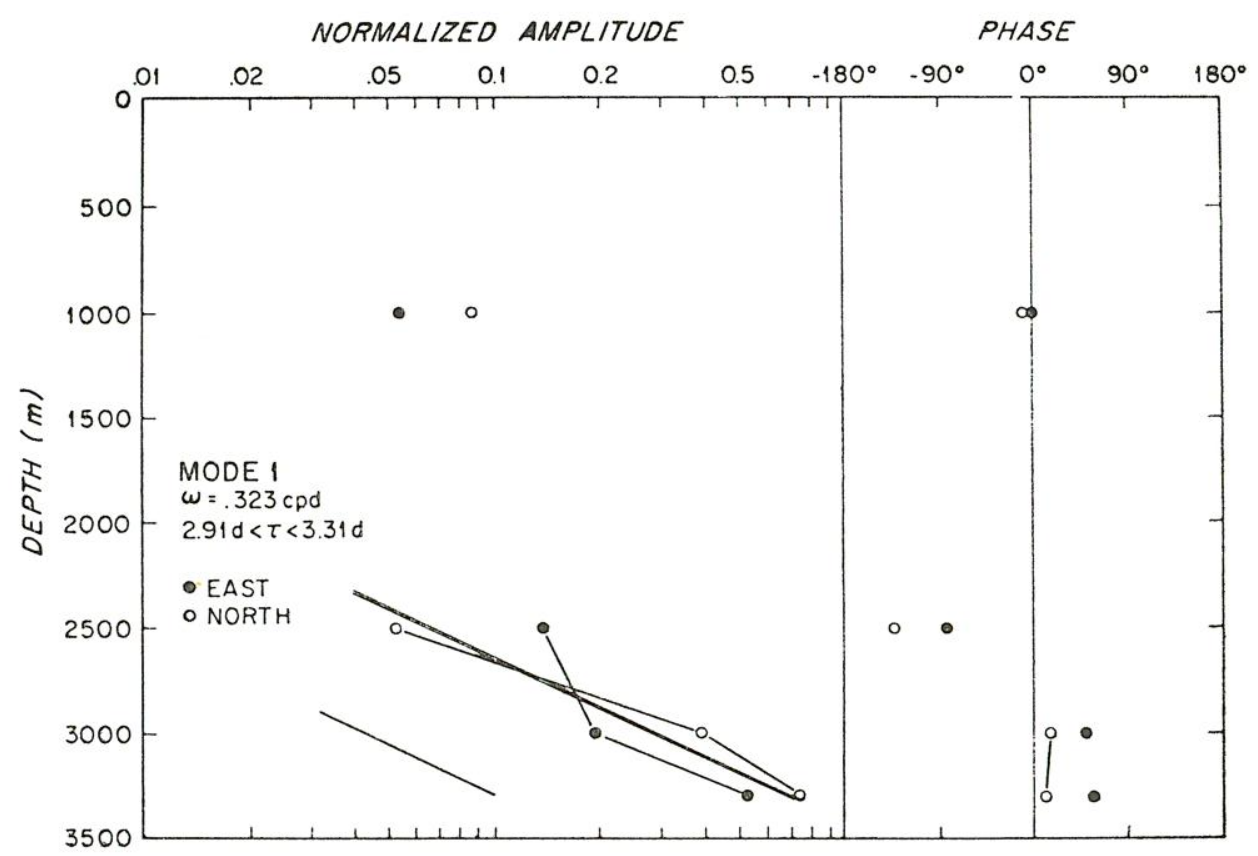

(e) 


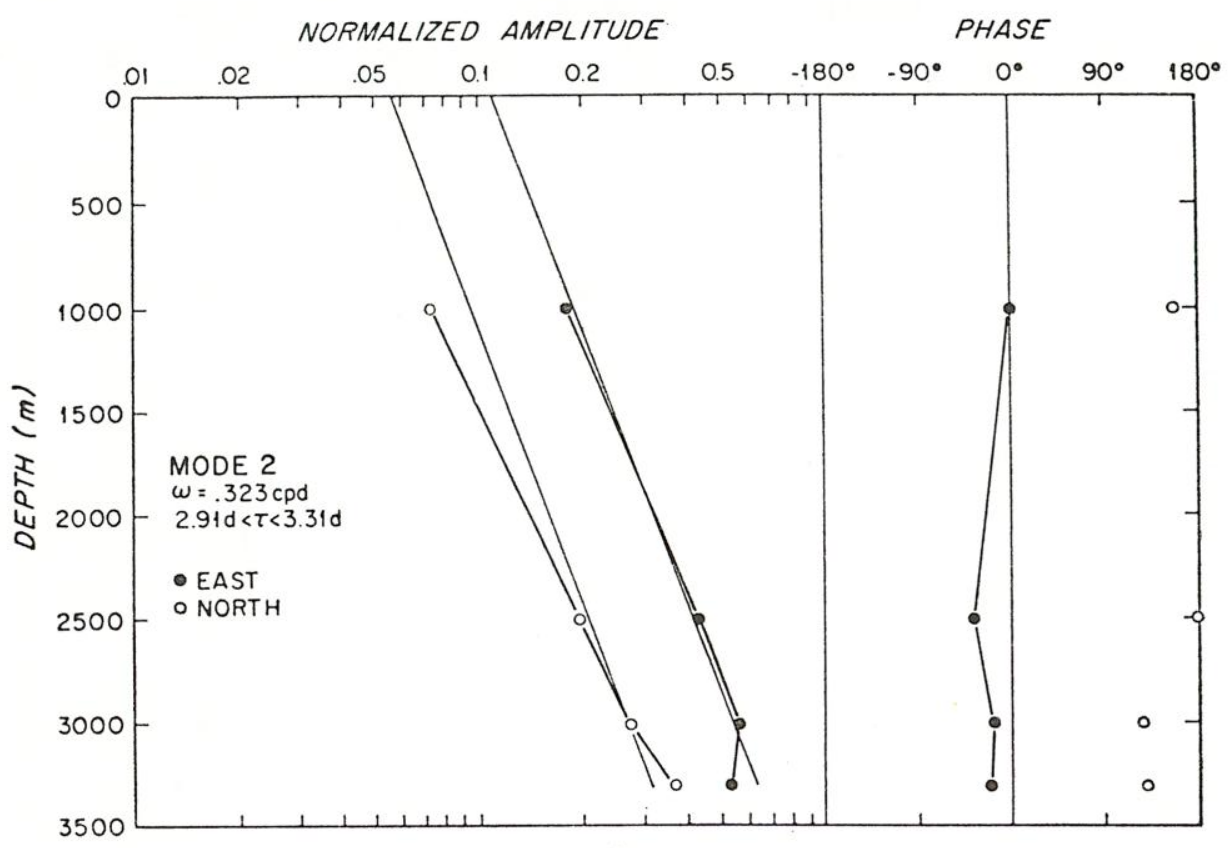

(f)

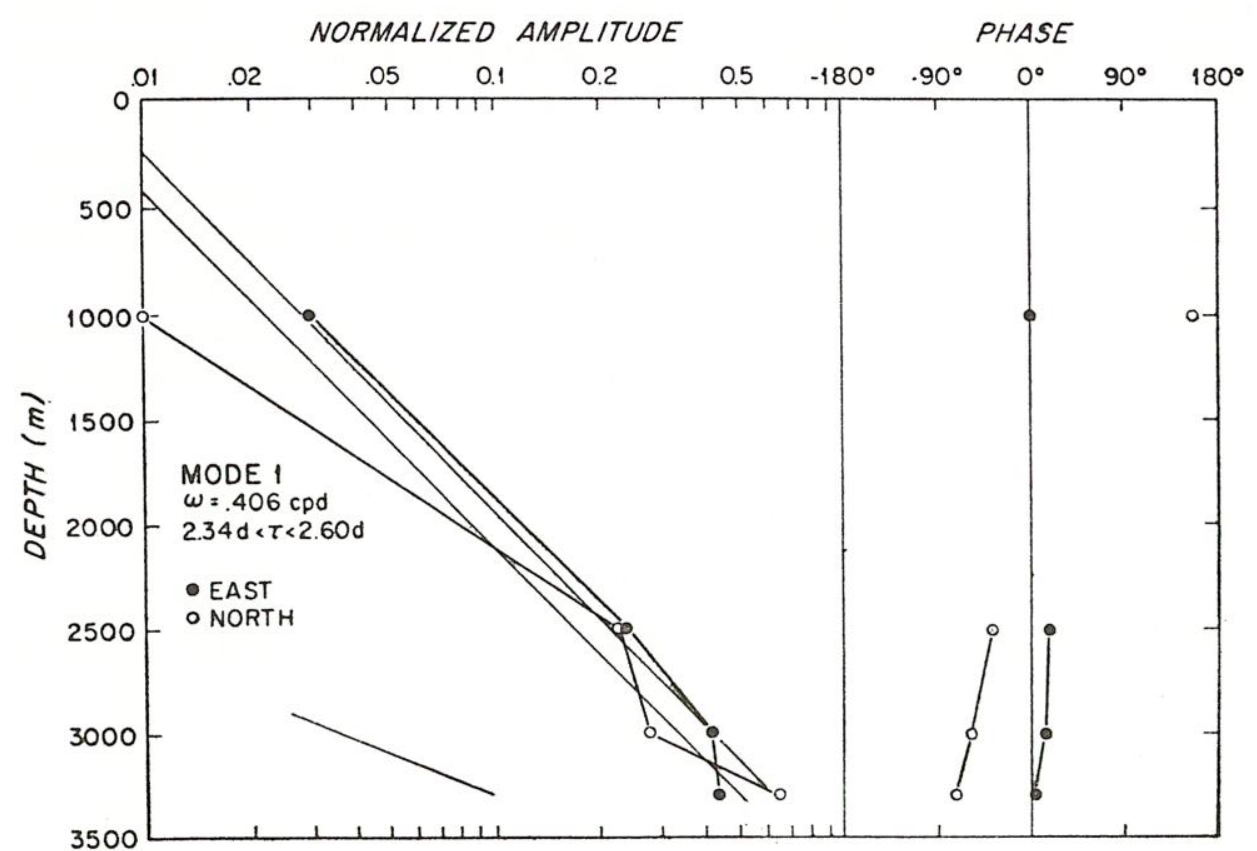

(g) 


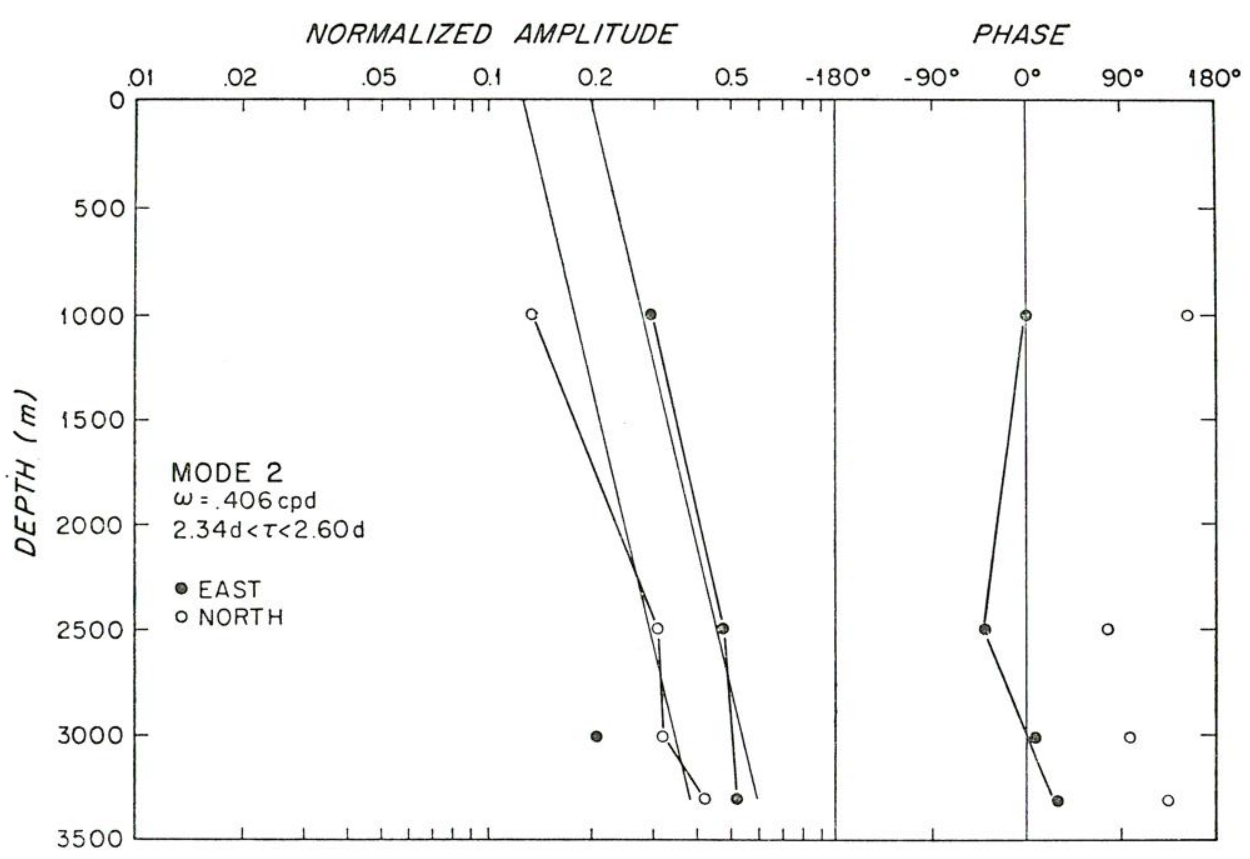

(h)

The phase lags between the eigenfunction and velocity components are shown in Figure 5, with only those having coherence amplitudes above the 95 percent confidence level being joined by lines. Phase is never determined with confidence throughout the water column: for the depth intensifying modes coherence increases with depth while the depth decreasing mode has significant coherence only at the shallowest depth. However, these phases vary only slowly with depth. Phase differences between $u$ and $v$, shown in Figure 6 at the various depths, also indicate that trends with depth and frequency are gradual in spite of the low coherence amplitudes.

In Figure 5, except for the second mode at the lowest estimate, velocity amplitudes increase approximately linearly with depth on the semi-logarithmic scale and the dashed lines give least squares fits according to

and

$$
u=U e^{-\lambda z} \text { or } \ln u=\ln U-\lambda z
$$

$$
v=V e^{-\lambda z} \quad \text { or } \ln v=\ln V-\lambda z .
$$

Minimizing the square error with respect to the fitting parameters $U, V$ it can be shown that

$$
E\left(\frac{V}{U}\right)=\left\{\frac{\prod_{i=1}^{N}}{\prod_{i}} v_{i=1} u_{i}^{1 / N}\right.
$$




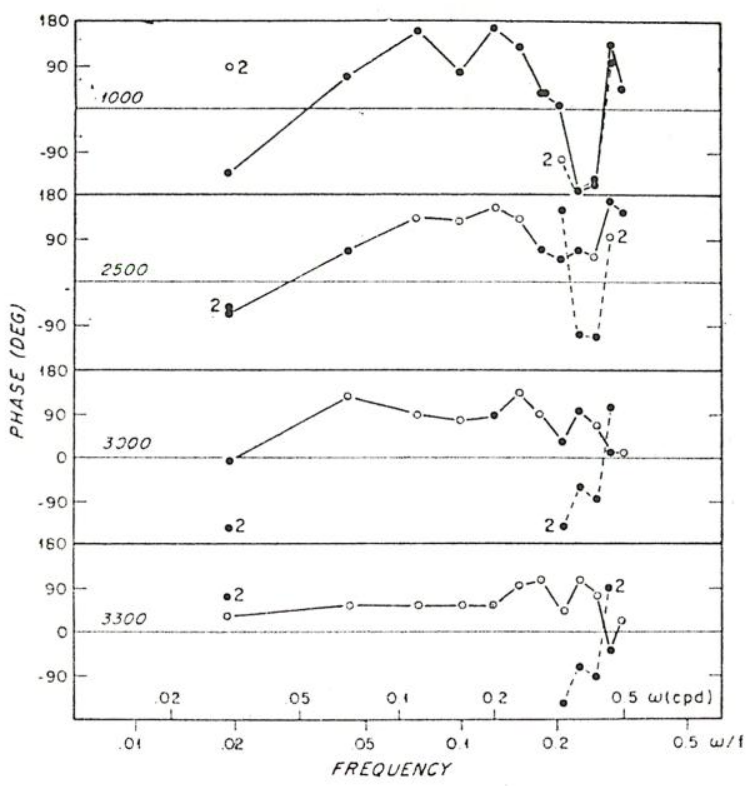

Figure 6. Phase difference between velocity components of the empirical modes with positive values meaning v leads $\mathrm{u}$. Open dots indicate estimates which are significant at the $95 \%$ level while the number 2 refers to the second mode.

where $N$ is the number of depth levels. Normalizing the $u$ amplitudes by the above ratio the profiles can be least squares fitted with two values at each depth using $V$ and $\lambda$ as fitting parameters. For eight points we are left with 5 degrees of freedom.

In several cases less than eight points were actually used. These included situations in which one point was far from the line and decreased the correlation of the fit to a value that was not distinguishable from zero. Most significantly it included cases just above $\omega=.28$ c.p.d. where the first mode was so highly bottom intensified that values at $1000 \mathrm{~m}$ fell very far from the line fit to the bottom three levels.

Correlation coefficients and type of fit are given in Figure 7a. On the right-hand side are shown the 90 percent confidence levels for the various numbers of points used in the fit. In almost all cases the correlation coefficient is significantly nonzero.

In Figure $7 \mathrm{~b}$ are shown the values of the normalized decay rate, $\lambda H$, versus frequency. A gradual increase from $\lambda H \cong 1$ to $\lambda H \cong 2$ occurs with frequency until $\omega$ $=.28$ c.p.d. above which the decay scale for mode 1 jumps to $\lambda H \cong 8$ (height scale of about $400 \mathrm{~m}$ ) before dropping again. The decay scale for mode 2 remains small.

The amplitude ratio $V / U$ in Figure 7 c shows a definite trend starting with low values (dominant $u$ component) at low frequencies and tending toward equipartition at higher frequencies.

There are also strong upslope variations in the distribution of energy with frequency. Figure 8 shows conventional log-log spectra of east and north components 


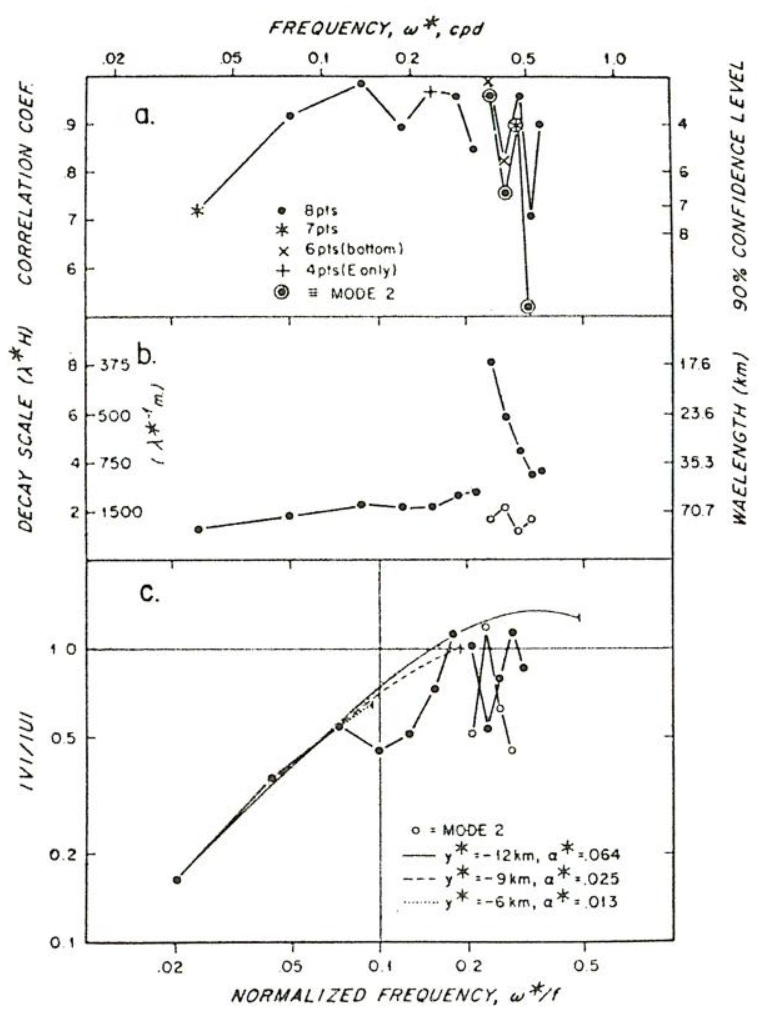

Figure 7. (a) Correlation coefficient for the least squares fits shown in Figure 5. Symbols refer to number of points used in the fit (see Figure 5 and text).

(b) Vertical decay scale computed from the straight line fit.

(c) Ratio of velocity components determined by the least squares fitting procedure versus frequency. This also gives a comparison of predicted ratio of velocity amplitude versus observed for the odd cross-slope mode interpretation and a constant horizontal wavenumber. For various indicated slopes we have used values of $y^{*}$ which give a best (eyeball) fit.

at moorings 571 and 572 . Over a distance of just $10 \mathrm{~km}$ at 3000 depth energy content below 0.1 c.p.d. decreases by more than an order of magnitude going north up the slope.

Another illustration of the strong dynamical constraint imposed by the bottom bathymetry is shown in Figure 9a which contains plots of the coherence amplitude and phase for the deepest current meters. Amplitudes are above the 95 percent confidence level for most of the frequency range and phases are remarkably different at the two moorings. At 571 the velocity components are out of phase throughout the low frequency band, but at 572 very low frequency motions are approximately in phase and change toward quadrature at higher frequencies with north leading east (clockwise rotation). At the shallow level (Figure 9b) coherence amplitudes are generally below the significance level although phases change in a uniform manner. 


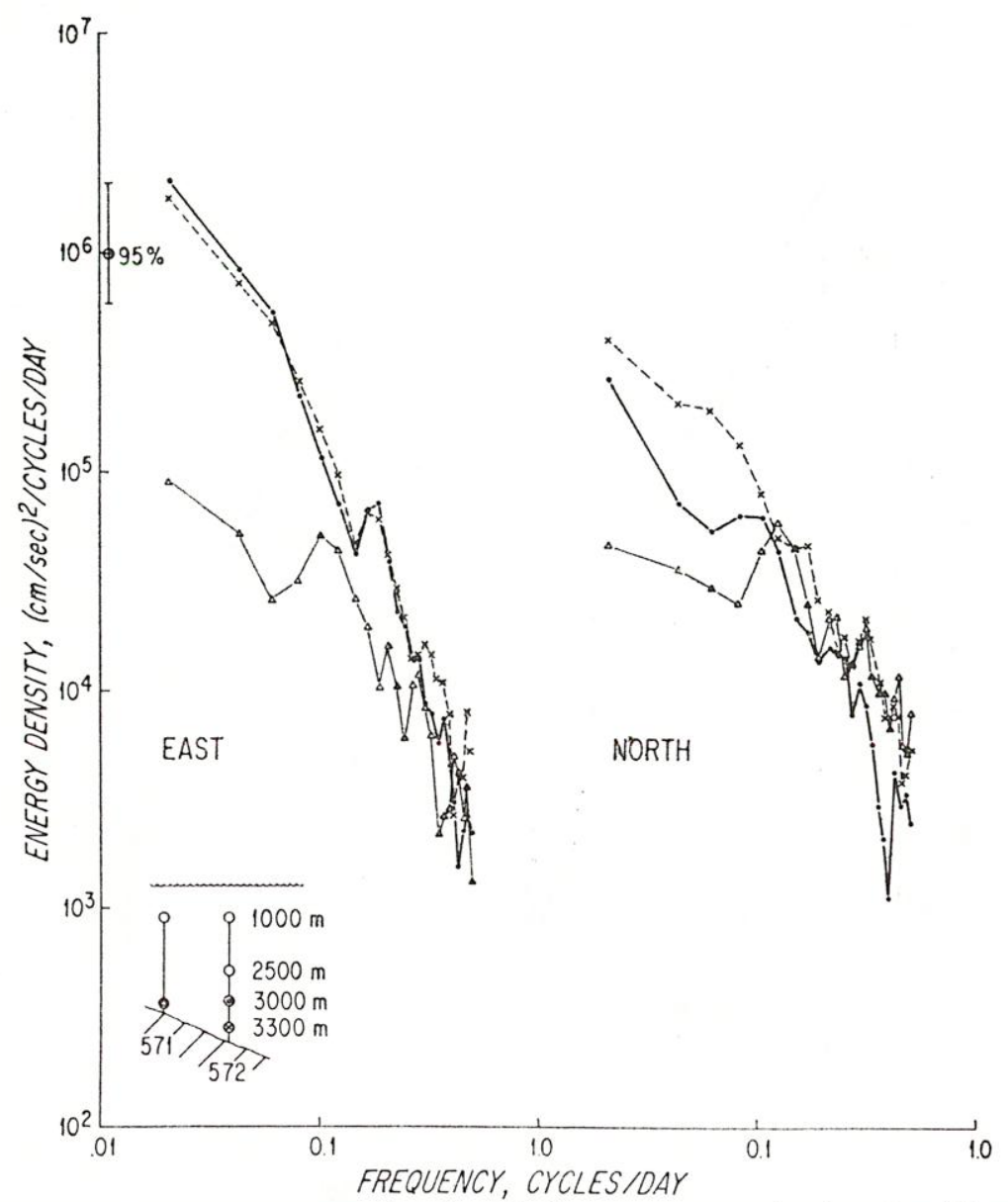

Figure 8. Variance versus frequency for the velocity components showing the differences between moorings. (a) Deep, (b) Shallow. (Continued on next page).

In summary, the low frequency motions can be divided into three frequency bands. Throughout, the most energetic empirical mode exponentially increases in amplitude toward the bottom. The degree of bottom intensification and ratio of north to east velocity components increase with frequency until $\omega=.28$ c.p.d. where two bottom trapped modes appear, one very highly trapped and the second less energetic one more weakly trapped. At very longest periods (19-96 days) a second mode is apparently significant and decreases in amplitude toward the sea floor with energy equally partitioned between velocity components.

Along with this vertical variability, the observed properties of the time-dependent motions are a strong function of position on the north slope. Moving a short distance north, the energy decreases by an order of magnitude and the velocity polarization changes radically below $1000 \mathrm{~m}$ depth. 


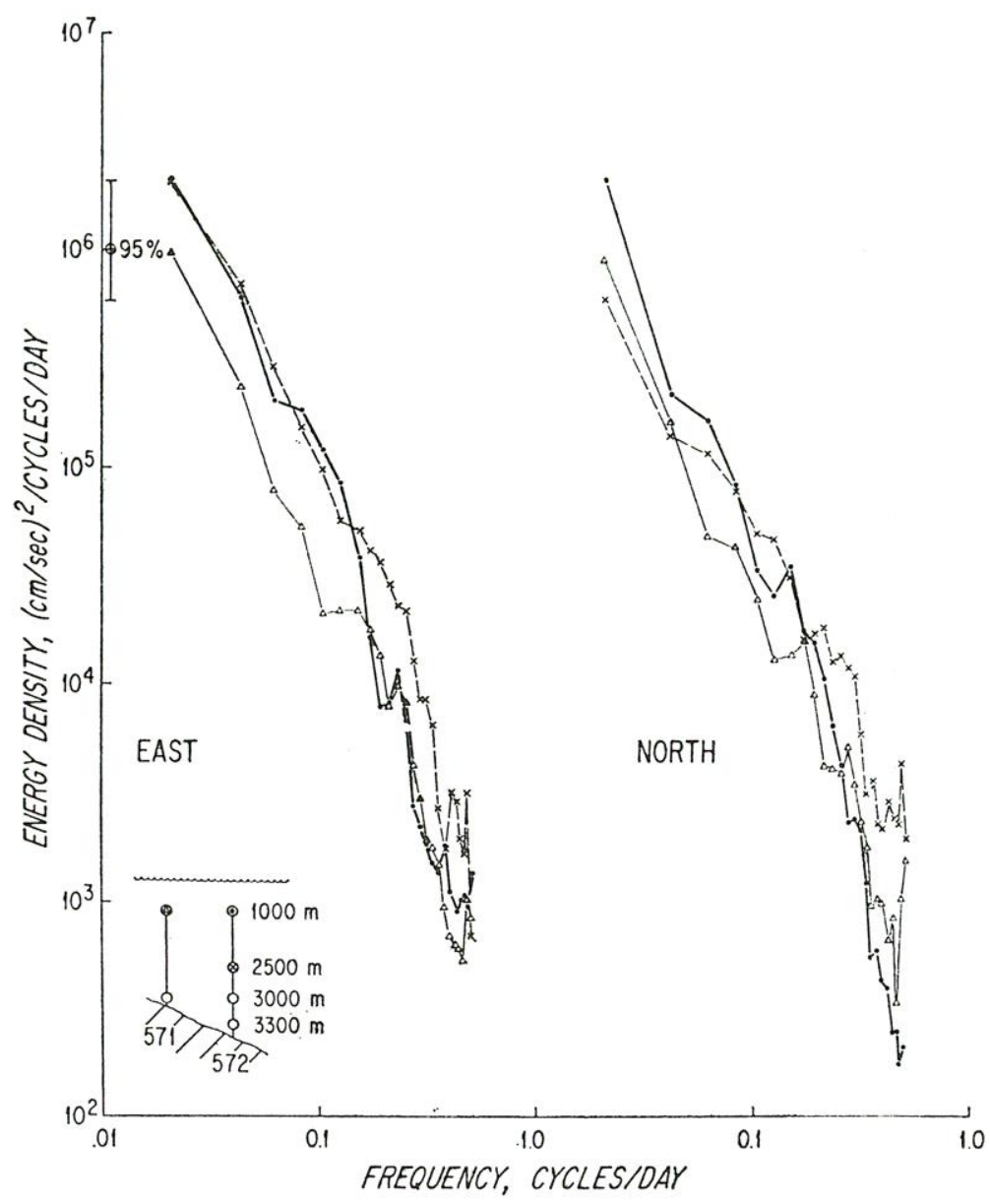

Figure 8 continued

\section{Interpretation}

a. Formulation. Assuming quasigeostrophic motions with length scales being significant fractions of the earth's radius, the inviscid vorticity conservation equation becomes (* is used throughout to denote a dimensional quantity)

$$
\frac{d \zeta^{*}}{d t^{*}}+\beta^{*} v^{*}=f_{0} w^{*} z^{*}
$$

where $d / d t^{*}=\partial / \partial t^{*}+u^{*} \cdot \nabla^{*}$ and $f=f_{0}+\beta^{*} y^{*}$. Including the time rate of change term in the heat equation (1), $w^{*} z^{*}$ can be eliminated to give

$$
\frac{d}{d t^{*}}\left[\zeta^{*}+\frac{\partial}{\partial z^{*}}\left(\frac{f_{0}}{\bar{T}^{*} z^{*}} T^{*}\right)\right]+\beta^{*} v^{*}=0 .
$$

We now define a length scale, $L$, determined by a wave scale or topographic scale, 

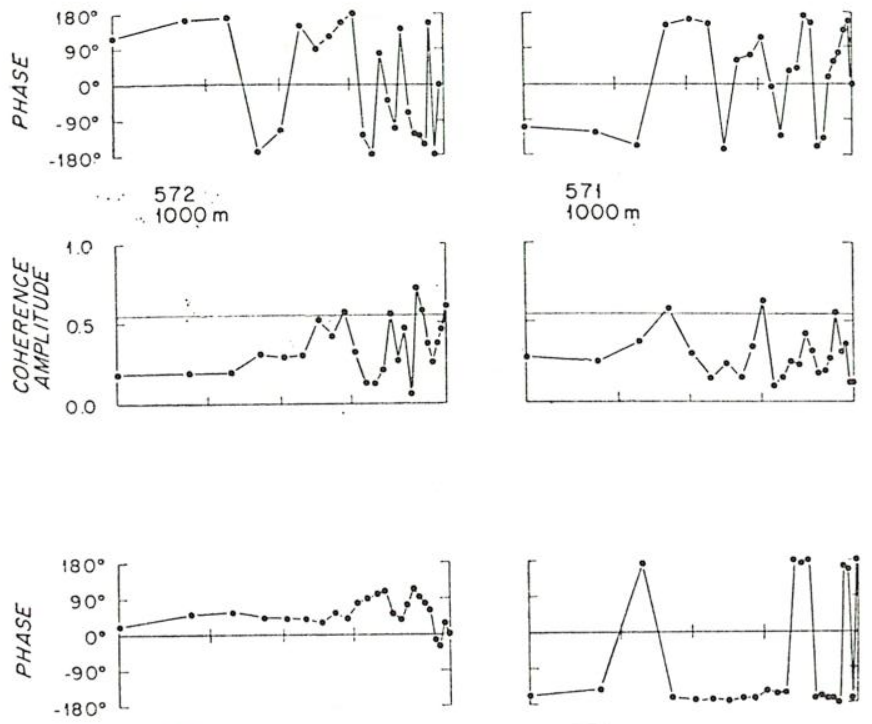

572
$3300 \mathrm{~m}$
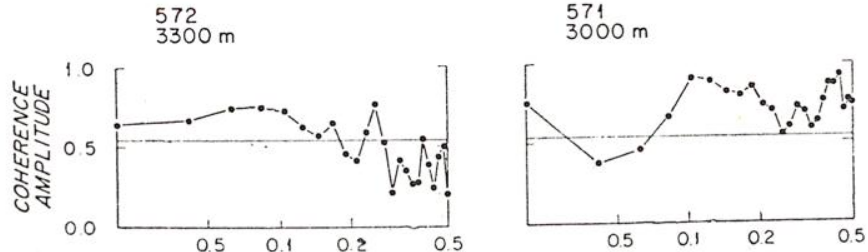

FREQUENCY (CPd)

Figure 9. Coherence amplitude and phase between velocity components at (a) deep (50 m off bottom), and (b) shallow (1000 m beneath surface) levels at moorings 571 and 572 . The 95 percent confidence level for amplitude is 0.52 .

a time scale $\omega^{*-1}$ from the wave frequency $\omega^{*}$, an amplitude scale $U$ and depth scale $H$ from the ocean depth. Four important parameters arise:

$$
\begin{aligned}
\epsilon & =\frac{U}{f_{0} L}, \text { the advective Rossby number } \\
\epsilon_{T} & =\frac{\omega^{*}}{f_{0}}, \text { the temporal Rossby number } \\
\beta & =\frac{\beta^{*} L}{f_{0}}, \text { a scaled } \beta \text { parameter }
\end{aligned}
$$

and

$$
B(z)=\frac{\left(g \alpha_{T} \bar{T}_{z^{*}}^{* 1 / 2} H\right.}{f L}=\frac{N(z) H}{f L}, \text { a stratification parameter. }
$$

Making use of geostrophy it is possible to rewrite (9) in nondimensional form for pressure alone: 


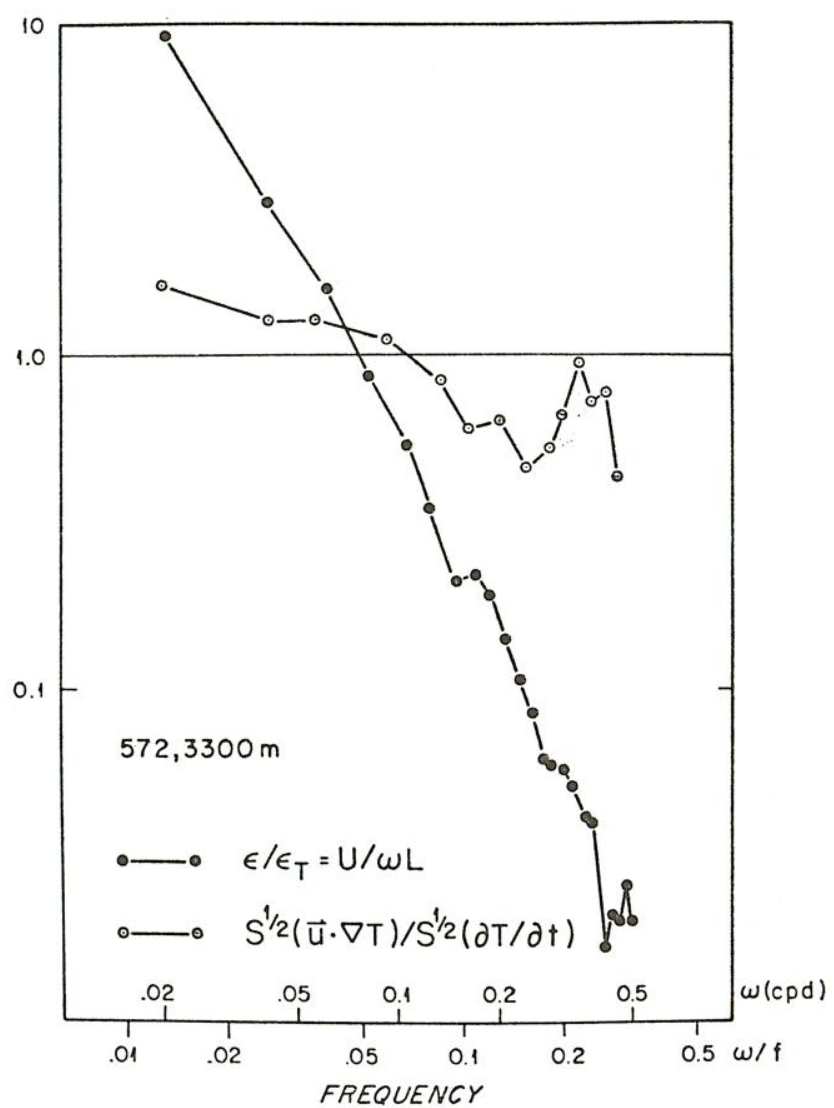

Figure 10. (a) Estimates of the ratio of horizontal advection to time rate of change from two methods (see text).

$$
\left(\epsilon_{T} \frac{\partial}{\partial t}+\epsilon \vec{u}_{h} \cdot \nabla_{h}\right)\left[\nabla_{h}^{2} p+\frac{\partial}{\partial z}\left(\frac{p_{z}}{B^{2}}\right)\right]+\beta p_{x}=0
$$

Motions are constrained to follow surface (assumed rigid and flat) and bottom $\left(z^{*}=-H+h^{*}\left(x^{*}, y^{*}\right)\right)$ boundaries. Therefore:

$$
p_{z}=0 @ \quad \text { @ }=0
$$

and

$$
\left(\epsilon_{T} \frac{\partial}{\partial t}+\overrightarrow{\epsilon u}_{h} \cdot \nabla_{h}\right) p_{z}=-B^{2}(z)\left(h_{x} p_{y}-h_{y} p_{x}\right) \quad @ \quad z=-1+h(x, y)
$$

b. Nonlinear effects. One measure of the importance of nonlinearities is given by the ratio of the advective to temporal Rossby numbers $\epsilon / \epsilon_{T}=U / L \omega^{*}$. If the smallest important length scale is that associated with the corrugations on the north wall of CGFZ, then this ratio can be estimated from the current meter data. From inspec- 

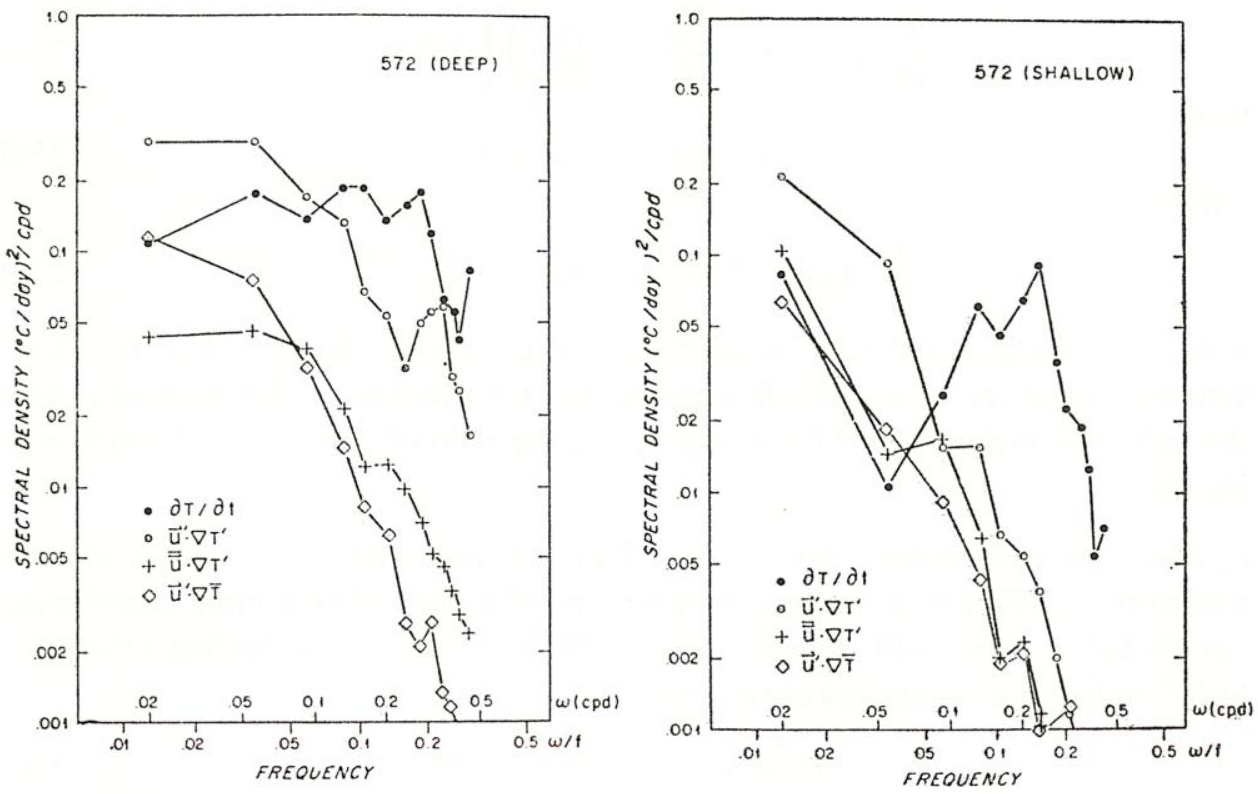

(b) Variance in the various contributions to the substantial derivative of temperature at mooring $572,3300 \mathrm{~m}$.

(c) As in $13 \mathrm{~b}$ but at $2500 \mathrm{~m}$.

tion of Figure 2 we choose $L \sim 18 / 2 \pi \mathrm{km}$ and plot in Figure 10a $\epsilon / \epsilon_{T}$ versus frequency for the deep instrument on 572 . The ratio drops to less than unity above about 0.07 c.p.d. (periods less than $\sim 14$ days).

The ratio of the advective to temporal derivative can be assessed without assumption about length scale from the heat equation. If we write temperature and velocity as a sum of means plus fluctuations $\left(T=T+T^{\prime}, \vec{u}_{h}=\overrightarrow{\bar{u}}_{h}+\vec{u}_{h}{ }^{\prime}\right)$ and use geostrophy to relate horizontal temperature gradients and vertical velocity gradients, then:

$$
\begin{aligned}
& \quad \frac{\partial T^{\prime}}{\partial t}+\frac{f}{g \alpha_{T}}\left(\bar{u} v_{z}-\bar{v} u_{z}{ }^{\prime}\right)+\left(u^{\prime} \bar{v}_{z}-v^{\prime} \bar{u}_{z}\right) \\
& +\left(u^{\prime} v_{z}{ }^{\prime}-v^{\prime} u_{z}{ }^{\prime}-\overline{u^{\prime} v_{z}{ }^{\prime}}+\overline{\left.v^{\prime} u_{z}\right)}+T_{z} w^{\prime}=0 .\right.
\end{aligned}
$$

In Figures 10b (deep) and 10c (shallow) the power spectra of the various terms in (12) are shown. The spectrum of the $\partial T^{\prime} / \partial t$ term is estimated from the temperature series at that depth whereas the vertical velocity gradients are differences between that depth and the one above. In Figure 10a we also plot the ratio of the amplitude of the principal advective term to the $\partial T / \partial t$ term; although it varies less than $\epsilon / \epsilon_{T}$ this ratio also drops below unity at roughly 0.1 c.p.d. above which there is about a decade in frequency where the dynamical balance is predominantly linear. Neglecting advection Eqs. (10) and (11) become 
with

$$
\frac{\partial}{\partial t}\left[\nabla_{h}^{2} p+\frac{\partial}{\partial z}\left(\frac{p_{z}}{B^{2}}\right)\right]+\beta p_{x}=0,
$$

$$
p_{z}=0 \text { on } \quad z=0
$$

and

$$
\frac{\partial}{\partial t} p_{z}=+B^{2}\left(h_{x} p_{y}-h_{y} p_{x}\right) \quad \text { on } \quad z=-1
$$

which we investigate below for small topographic variation, $h(x, y)<<1$. (For convenience we set $\epsilon_{T}=1$ and will consider the relative size of the time derivative through the nondimensional frequency $\omega$, i.e., the ratio of dimensional frequency to inertial.)

c. Waves on a plane slope; $h(x, y)=\alpha y$. This is a crude attempt to model the north wall of the CGFZ and is a necessary first step before considering more complicated models below which will account for the effects of small scale bottom relief. We look for plane wave solutions of the form

$$
\overrightarrow{p(x, t)}=P(z) e^{i(k x+l y-\omega t)}
$$

where $P(z)$ is a vertical eigenfunction. Orthogonal modes, each with an associated dispersion relation, are:

$$
P(z)=A_{0} \cosh \lambda_{0} z
$$

provided tanh $\lambda_{0}=-\frac{\alpha}{\beta}\left(\frac{\lambda_{0}{ }^{2}-B^{2} K^{2}}{\lambda_{0}}\right)$ and $\omega=-\frac{B^{2} \alpha k}{\lambda_{0} \tanh \lambda_{0}}$ and

$$
P(z)=A_{n} \cos \gamma_{n} z, \quad n=1,2,3 \ldots
$$

provided $\tan \gamma_{n}=-\frac{\alpha}{\beta}\left(\frac{\gamma_{n}{ }^{2}+B^{2} K^{2}}{\gamma_{n}}\right)$ and $\omega=-\frac{\beta k B^{2}}{B^{2} K^{2}+\gamma_{n}{ }^{2}}$

with $K^{2}=k^{2}+l^{2}$ and arbitrary amplitudes $A_{0}$ and $A_{n}$.

These wave roots have been discussed previously by Rhines (1970) and Suarez (1971). The zeroth root is their topographic Rossby wave (TW) which increases in amplitude toward the bottom and has frequency proportional to bottom slope $\alpha$ while the infinite set $\left(\gamma_{n}, \cos \gamma_{n} z\right)$ are modified baroclinic Rossby waves (BW) whose frequencies are proportional to $\beta$. In the limit that $\alpha / \beta \rightarrow \infty$

so that the TW root becomes

$$
\lambda_{0} \rightarrow B K, \quad \text { and } \quad \gamma_{n} \rightarrow\left(\frac{2 n-1}{2}\right) \pi
$$

$$
P(z)=A_{0} \cosh B K z ; \quad \omega=-\frac{B \alpha k}{K \tanh B K}
$$

and the BW root is 


$$
P(z)=A_{n} \cos \left(\frac{2 n-1}{2}\right) \pi z ; \quad \omega=-\frac{\beta k B^{2}}{B K^{2}+\gamma_{n}^{2}}
$$

These free waves appear to have the potential of explaining the vertical distribution of $K E$ (Fig. 4 in SH) and the empirical orthogonal functions (Fig. 5). They are of two types: a topographic wave which is depth intensified and a set of baroclinic waves which have nodes at the bottom (i.e., intensify away from the bottom). Moreover for representative values of $B, \alpha$ and $\beta$ periods of order 100 days (our very low band) are very low frequency TWs but very high frequency BWs. The flow associated with the TW would be approximately east-west, along the bathymetry, while that of the BW is more north-south across the bathymetry consistent with Figures $5 \mathrm{a}, \mathrm{b}$ (periods 19.2-96 days).

However, there are some problems. (1) The dispersion relation (18b) predicts a maximum frequency $\omega_{\max }=\beta B / 2 \gamma_{n}\left(\beta^{*}(N / f) H /(2 n-1) \pi\right.$, dimensional frequency) when $\vec{k}=\left(-\frac{\gamma_{n}}{B}, 0\right)$ for BWs. Using $N \sim 12$ c.p.d., $f=1.6$ c.p.d., $H \sim 3$ $\mathrm{km}$ and $\beta^{*}=.00019$ c.p.d. $/ \mathrm{km}$ gives a shortest period of 739 days. $\dagger(2)$ It is not possible to explain the rapid drop in east and north variances as one moves north (upslope) between 572 and 571. (3) A further problem is that the waves, being transverse, give rise to in- or out-of-phase velocity components. Figures 5 and 6 indicate quadrature is a better approximation with $v$ leading $u$.

With respect to the time scale problem there are two possibilities. Nonlinearities are significant and may dominate the dynamics at frequencies less than 0.1 c.p.d. Low frequency motions in other parts of the North Atlantic, particularly the MODE region, exhibit some features of linear dynamics such as vertical structure (Richman et al., 1977) and westward phase propagation (McWilliams and Flierl, 1976) but, at the same time, the dynamical balance is strictly nonlinear (Bryden, 1976) and the time scale too short for freely propagating baroclinic waves (Richman et al., 1977). However, within the realms of linear dynamics there is a rationalization, described below, which also gives an explanation for the rapid upslope energy decrease and $90^{\circ}$ phase relationship between velocity components.

d. Waves in a smooth trough. The moored array is situated on the north wall of an east-west trough. A simple conceptual model might be that in Figure 11 in which energy reflection will occur at slope changes. We shall not pursue the problem in great detail here. Suarez (1971) has considered slightly different geometries and Rhines (1969) the related homogeneous case. Our interest is mainly in the vertical forms of the available eigenmodes and their frequency dependence.

For wave periods shorter than 96 days the only free waves are the barotropic Rossby waves in flat regions and topographic waves over slopes. These are matched

$\dagger$ Using more realistic stratification reduces this shortest period to 354 days, still sufficiently long so as to be below our lowest frequency band. 


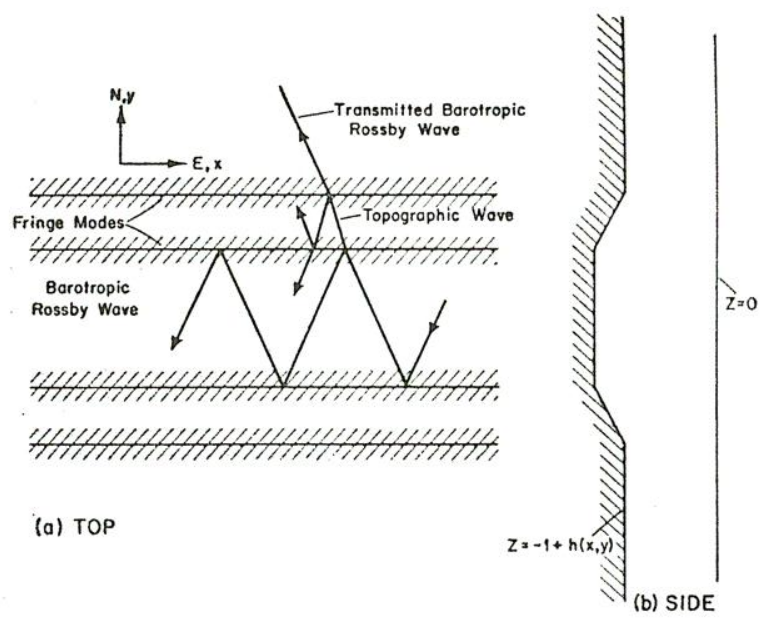

Figure 11. Conceptual model of CGFZ.

at slope changes by the baroclinic modes which must have imaginary upslope wavenumber components and decay away from the regions of changing slope. We shall call these "fringe modes."

Consider the reflection of a flat bottom barotropic wave with incident wavenumber $\vec{\kappa}_{i}$ at the edge of the north wall. Frequency and longslope wavenumber must be preserved so, from $(15 b)$ and $(16 b)$

$$
\lambda_{0} \tanh \lambda_{0}=-\gamma_{n} \tan \gamma_{n}=\left(B K_{i}\right)^{2}\left(\frac{\alpha}{\beta}\right) .
$$

For $\alpha / \beta>>1$, and waves that are short enough that $B K_{i} \sim 0(1)$ the TW root is $\lambda_{\theta}$ $\sim\left(B K_{i}\right)^{2} \alpha / \beta>>1$, highly bottom trapped, and the BW roots are $\gamma_{n} \sim \frac{2 n-1}{2}$ $\pi$, baroclinic fringe modes with nodes at the bottom. On the other hand very long waves with $\left(B K_{i}\right)^{2} \alpha / \beta \sim 0$ (1) possess solutions to (19) such that $\lambda_{0} \sim 0$ (1) and $\frac{2 n-1}{2} \pi<\gamma_{n}<n \pi$. As $\left(B K_{i}\right)^{2} \alpha / \beta \rightarrow 0, \lambda_{0} \rightarrow 0$ and $\gamma_{n} \rightarrow n \pi$. The TWs are more weakly bottom trapped and the fringe waves more closely resemble flat bottom modes.

The dependence of vertical structure on incident wavenumber is illustrated by Figure 12. Also shown is the maximum incident wavenumber allowed for each of the lowest frequency bands: only the very lowest band can excite baroclinic fringe modes with $\gamma_{1}$ near $\frac{\pi}{2}$ (node at the bottom). Higher bands have progressively larger $\gamma_{1}$.

Within any given frequency band we might guess that the incident wavefield was broadband in wavenumber. The shorter waves transmit upslope in the form of highly trapped TWs exciting fringe waves with nodes at the bottom while the longer 


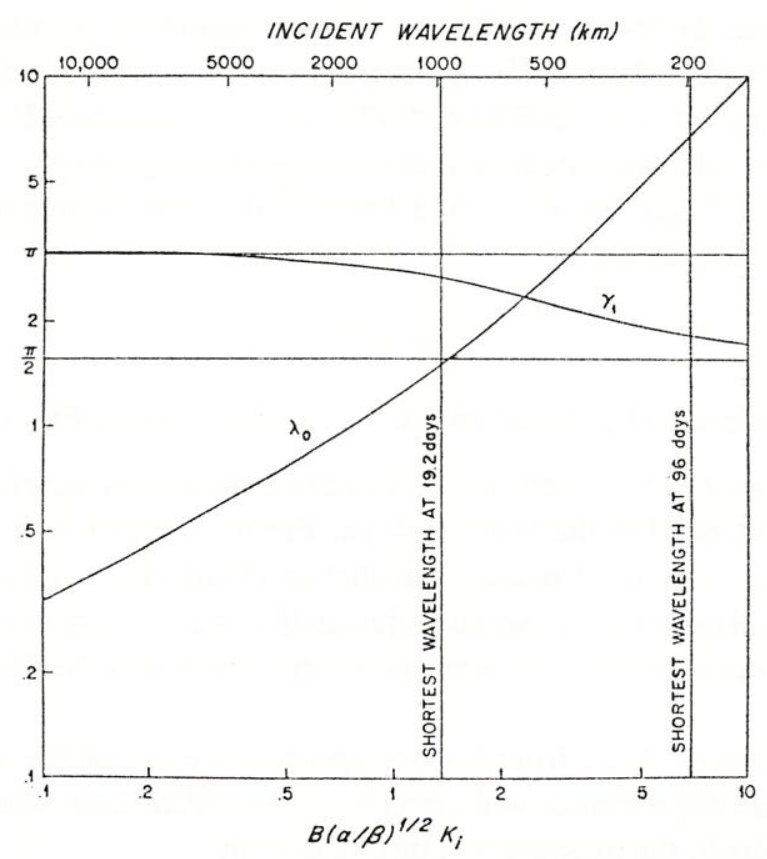

Figure 12. Eigenvalues, $\lambda_{0}$ and $\gamma_{1}$, for the topographic and first baroclinic modes versus incident wavenumber showing that only in the lowest band can there be forced a baroclinic mode with near bottom node.

ones transmit as more weakly bottom trapped TWs forcing fringe waves with nodes off the bottom. Suarez (1971) has shown that in the former case relatively more energy is transferred into the fringe modes because of the greater mismatch in vertical structure between incident and transmitted waves. Consequently, near our moorings the transmitted waves will be weakly bottom trapped and the fringe modes those with nodes nearer the bottom with only those in the lowest band having nodes essentially right at the bottom.

In order that fringe waves exist at these frequencies, the denominator in (16b) must nearly vanish so that $(l / k)^{2}=-\left(1+\gamma_{n}{ }^{2} / B^{2} k^{2}\right)$. Choosing the root that decays upslope gives

$$
\frac{v}{u}=-\frac{p_{x}}{p_{v}}=\frac{k}{l}=-i\left(1+\frac{\gamma_{n}^{2}}{B^{2} k^{2}}\right)^{-1 / 2}
$$

For the lowest fringe mode, $\gamma_{1} / B k \geqslant \gamma_{1} / B k_{i} \cong \lambda_{i} / 120(\mathrm{~km})$. According to Figure 12 the shortest allowed wavelength in the lowest band for the barotropic Rossby wave is about $200 \mathrm{~km}$ giving $\gamma_{1} / B k \geqslant 1.67$ and $|\nu / u| \leqslant .51$ : The longshore component should dominate upslope by at least a factor of two and lag in phase by $90^{\circ}$. Figures $5 \mathrm{~b}$ and 6 indicate that we have nearly the correct phase but have somewhat underestimated the amplitude ratio. 
Longer waves in the lowest band must have wavenumbers oriented more upslope. Taking $\lambda_{0_{k}}=1.2$ from Figure $7 \mathrm{~b}$, estimating $\alpha^{*}=.05$, and matching frequencies according to (19) gives $K_{i} \cong .0025 \mathrm{~km}^{-1}$ ( $2562 \mathrm{~km}$ wavelength). For a 19-day wave $k=.00099 \mathrm{~km}^{-1}$ : the wavenumber vector points virtually upslope. The transmitted TW with $B K=1.2$ has $l \cong K=.053 \mathrm{~km}^{-1}$ and points even more upslope. The ratio of velocity components is:

$$
\frac{v}{u}=+\left|\frac{k}{l}\right|=.019
$$

this time underestimated by almost an order of magnitude (see Fig. 7c).

e. Finite north wall. The amplitude ratio (21) depends on several environmental parameters-most notably the bottom slope. From the dispersion relation (18a) it can be seen that $k \propto 1 / \alpha$. However, a reduction of the effective bottom slope sufficient to force agreement in (21) seems unjustifiable and, furthermore, the plane wave solutions are inconsistent with the upslope energy decay and the $90^{\circ}$ phase between $v$ and $u$.

If energy is reflected back from further upslope (the top of the north wall in our model) then cross-slope modes will develop. In the ideal case where energy is reflected at one isobath, the pressure will be of the form

giving

$$
\overrightarrow{p(x, t)}=e^{i(k x-\omega t)} \cosh \lambda_{\diamond} z \times\left\{\begin{array}{ll}
\sin l y \\
\cos l y
\end{array}\right\} \text { odd }
$$

$$
\frac{v}{u}=-\frac{k}{l} \times\left\{\begin{array}{c}
\tan l y \\
-\cot l y
\end{array}\right\} \begin{aligned}
& \text { odd } \\
& \text { even }
\end{aligned}
$$

where $k$ and $l$ are related through the dispersion relation (18a) and $y$ is now measured from the center of the step. Existence of modal rather than plane wave structure is consistent with a $90^{\circ}$ phase shift between $u$ and $v$. $v$ leads $u$ where the observed phases are significant, suggesting odd modes for $\tan l y<0$ and even modes for cot $l y<0$. Favoring odd modes and, therefore, $|l y|<\frac{\pi}{2}$ is the fact that as $\omega \rightarrow B \alpha,|k| \rightarrow \kappa$ and $l \rightarrow 0$ so that $v / u$ remains finite only in this case.

The ratio in (23) depends on two imprecisely determined environmental parameters, the distance $y$ from midslope and, through the dispersion relation, the bottom slope $\alpha$. The best eyeball fit of (23) is plotted on Figure 7c using different values for $\alpha^{*}$ and $y^{*}$. Although a reasonable fit can be obtained, $\alpha^{*}$ must be greater than about .04 in order that such waves exist throughout the band, a value close to our initial estimate.

f. Small scale roughness. The fringe modes have negligible amplitude at the bottom and will be little affected by the small scale roughness of the north wall. On the 
other hand, the topographic waves have their maximum amplitudes on the bottom. This subsection briefly considers the effect of an idealized form of the roughness in the framework of perturbations to a long topographic wave produced by shorter corrugations on a plane slope. The problem is similar to that treated by Suarez (1971) and McWilliams (1974) (both stratified) and Rhines and Bretherton (1973) (homogeneous) except that the corrugations will be aligned in the upslope direction rather than alongslope.

Consider the effect of sinusoidal isobaths represented in dimensional form by $\dagger$

$$
z^{*}=-H+\alpha^{*} y^{*}+\epsilon^{*} \sin \lambda x^{*} .
$$

The topographic wavenumber $\lambda$ provides a convenient length scale for scaling: we redefine $B=\left(\frac{N}{f}\right) H \lambda$ and $\alpha=\alpha^{*} / \lambda H$ and set $\epsilon=\epsilon^{*} / H$. Using $\lambda=2 \pi / 18$ $\mathrm{km}^{-1}, N / f=7.5, \alpha^{*} \cong 0.05, \epsilon^{*}=400 \mathrm{~m}$, and $H=3 \mathrm{~km}$ gives $B=7.9, \alpha=0.05$, and $\epsilon=0.13$. We assume that $\alpha<<1, \epsilon<<1$, and $\beta^{*}=0$ so that our attention is focused on topographic wave dynamics. Frequency is renormalized by the maximum topographic wave frequency for shortwaves, $N \alpha^{*}$, i.e.

$$
\sigma=\omega / B \alpha=\omega^{*} / N \alpha^{*}
$$

With coefficients depending only on $x$ we can write

$$
\overrightarrow{p(x, t)}=e^{i(l y-\sigma t)} P(x, z)
$$

and Eqs. (13a-c) become:

with

$$
\left(\frac{\partial}{\partial x^{2}}+\frac{1}{B^{2}} \frac{\partial}{\partial z^{2}}-l^{2}\right) P=0,
$$

and

$$
P_{s}=0 \text { on } z=0
$$

$$
i \sigma P_{z}=B\left[P_{x}-i\left(\frac{l \epsilon}{\alpha}\right) \cos (x) P\right] \text { on } z=-1 .
$$

In the problem posed above there are two inherent length scales, one imposed on the flow by the small corrugation scale $\lambda^{-1}$ and the other (assumed) larger scale $l^{-1}$ of the wave field. Using $l$ as a small parameter (27) is solved in the Appendix using a two-scale procedure under the assumption that $l \epsilon / \alpha \sim 0\left(l^{1 / 2}\right)$, the highest order at which we have been able to make progress. Our observations have indicated $\epsilon / \alpha$ $\sim 0.13 / .05=2.6$ while if we take the vertical decay scale $\lambda=2$ as typical $|l| \leqslant$ $\frac{\lambda f}{N H} \cdot \frac{18}{2 \pi}=.27$ so that $|l|(\epsilon / \alpha)<0.7$ while $|l|^{1 / 2} \epsilon / \alpha<1.4$. At lowest or-

$\dagger$ As long as the corrugations vary on a small length scale in only one direction their form can be arbitrary and the results expressed in Fourier transforms. We choose this explicit form for convenience and clarity. 


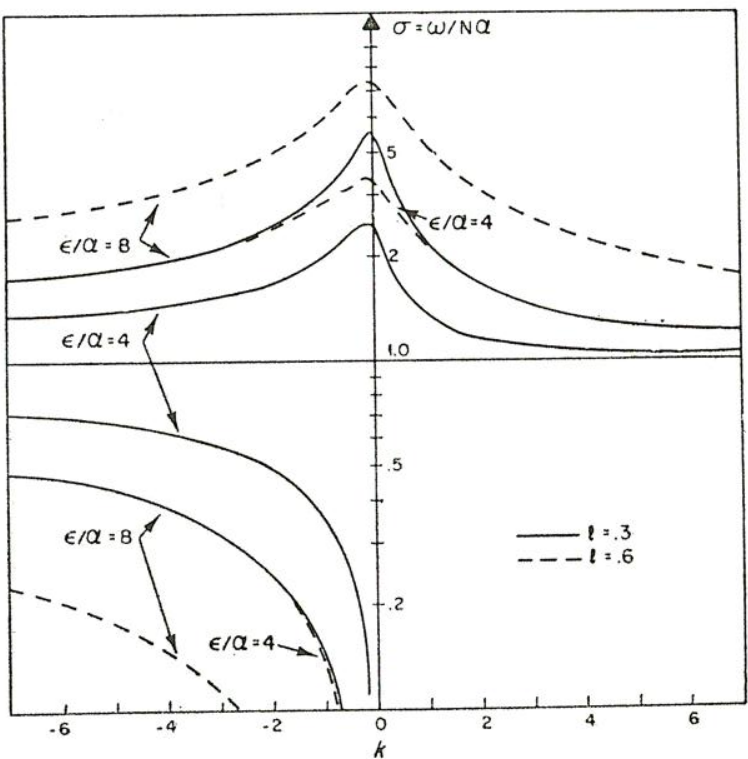

Figure 13. Dispersion relation for topographic Rossby waves over corrugations of two different amplitudes $(\epsilon / \alpha)$ and two different north-south wavenumbers $(l)$ for $K=0.33$.

der the $|l| \epsilon / \alpha \sim 0(l)$ case can be recovered from $|l|^{1 / 2} \epsilon / \alpha \sim 0(1)$ by letting $\epsilon \rightarrow 0$. We find that the lowest order dispersion relation is

$$
\sigma=-\frac{k}{K \tanh B K}+\left(\frac{\epsilon}{\alpha}\right)^{2} \cdot \frac{l^{2}}{2 \tanh B K} \cdot \frac{\sigma}{\sigma^{2}-1}
$$

and the first two orders of the pressure expansion are

$$
P(x, z)=e^{i K \varpi}\left\{\frac{\cosh B K z}{\cosh B K}+\left(l \frac{\epsilon}{\alpha}\right) \frac{1}{\sigma^{2}-1}(\sigma \cos x+i \sin x) \frac{\cosh B z}{\cosh B}+0(l)\right\} .
$$

Equation (28) is cubic in $\sigma$. Taking roots with $\sigma>0$ we plot in Figure 13 the dispersion relation for $K=0.33(B K=2.6)$ and two values of the ratio $\epsilon / \alpha$. There are 2 branches, a plane slope topographic wave when $\sigma<1$ that is modified by the corrugations and two oppositely traveling corrugation waves for $\sigma>1 \dagger$ that are modified by the plane slope. As $\epsilon / \alpha$ becomes small the region above $\sigma>1$ in which wave motions are allowed also becomes small and in the limit $\epsilon / \alpha \rightarrow 0$ only the plane slope wave is allowed. Equation (29) describes a weakly bottom trapped wave at lowest order with $e$-folding scale $(B K)^{-1}$ and corrugation scale perturbations at $0\left(l^{1 / 2}\right)$ decaying into the interior at the more rapid rate $B^{-1}$.

$\dagger$ Actually there is a small gap between $1<\sigma<1 / \tanh B K$ in which only the corrugation wave traveling in the positive $x$ direction is allowed. 


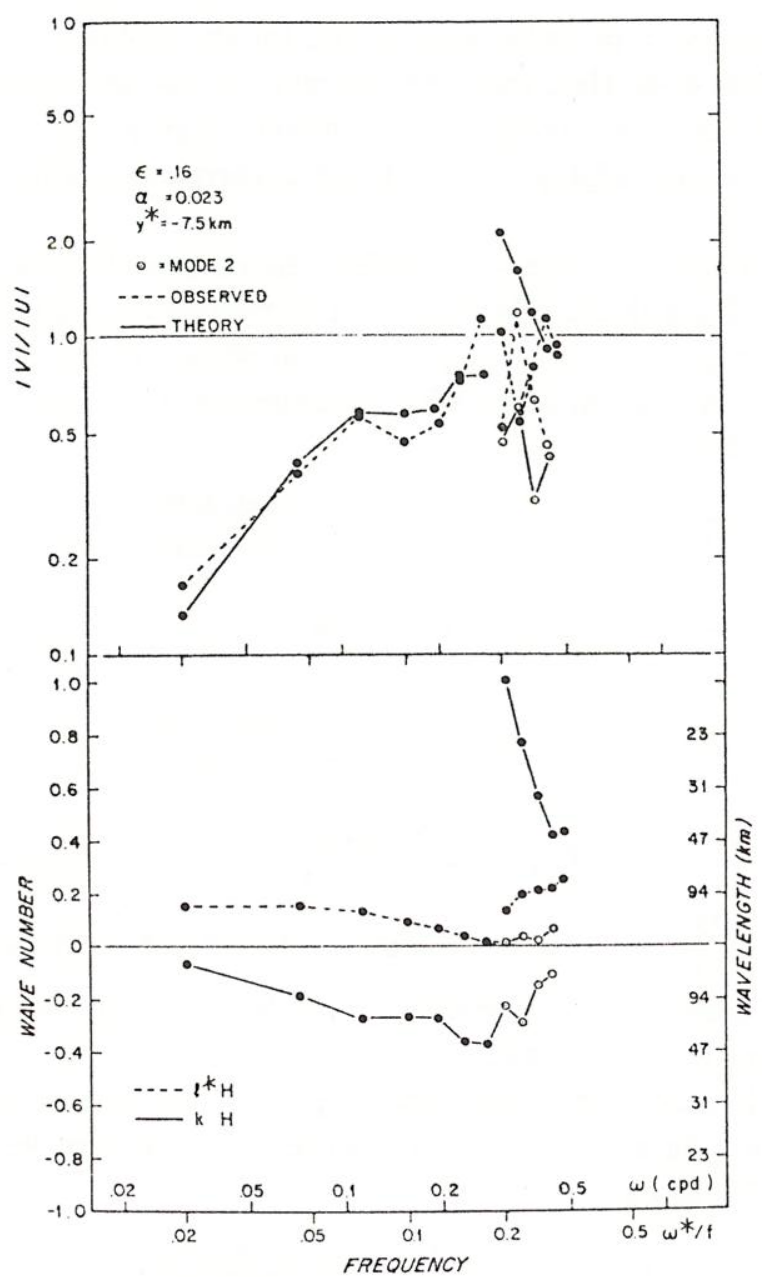

Figure 14. Comparison of the predicted ratio of velocity amplitudes versus observed (Figure 7c) for the odd cross slope mode using $\alpha=.023$ and a horizontal wavenumber computed from the vertical decay scale in Figure $7 \mathrm{~b}$. In the bottom plot are shown computed values of the horizontal wavenumber components.

In Eq. (29) there is an apparent singularity at the transition frequency $\sigma=1$. It can be shown that $\frac{l \epsilon}{\alpha} \frac{1}{\sigma^{2}-1} \rightarrow|k|^{1 / 2}$ when $k \rightarrow-K$ whereas $\frac{l \epsilon}{\alpha} \frac{1}{\sigma^{2}-1}$ $\rightarrow\left(2 \frac{K}{|\sigma-1|}\right)^{1 / 2} \quad$ when $k \rightarrow K$. Our procedure is valid for the westward propagating roots at $\sigma=1$ but breaks down for the eastward ones for which the amplitude of the corrugation scale disturbance becomes comparable to the amplitude of the larger scale wave.

If we identify $\omega^{*}=0.28$ c.p.d. $(\omega=.18)$ with this transition frequency then 
corrugation effects give a plausible explanation for the motions existing above this frequency in Figures 4e-h. Here there are two wave roots-one traveling to the west looking much like the lower frequency topographic waves and a second traveling to the east in which the highly bottom trapped corrugation effect is much more pronounced.

Previously we found it necessary to consider the north wall to be finite and introduced upslope modes with the odd mode best explaining the frequency dependence of $v / u$. With corrugations a good approximation should be obtained by summing waves traveling up and down slope with wavenumbers $(k, l)$ and $(k,-l)$. To $0\left(l^{1 / 2}\right)$ one obtains from (29):

$$
\text { odd } \begin{aligned}
P_{0}(x, z)= & e^{i(k x-\omega t)}\left\{\sin l y\left(\frac{\cosh K B z}{\cosh K B}\right)+\right. \\
& -\left(l \frac{\epsilon}{\alpha}\right) \frac{\cos l y}{\sigma^{2}-1}(i \sigma \cos x+\sin x) \frac{\cosh B z}{\cosh B} \\
\text { even } \quad P_{e}(x, z)= & e^{i(k x-\omega t)}\left\{\cos l y\left(\frac{\cosh K B z}{\cosh B}\right)+\right. \\
& \left(l \frac{\epsilon}{\alpha}\right) \frac{\sin l y}{\sigma^{2}-1}(i \sigma \cos x+\sin x) \frac{\cosh B z}{\cosh B} .
\end{aligned}
$$

$\alpha^{*}=\frac{\omega^{*}}{N}=\frac{.28}{12}=.023$ and using $\epsilon=.16\left(\epsilon^{*} / \alpha^{*}=6\right)$ we show in Figure 14 the calculated ratio $|v| /|u|$ for various $y$ and the observed values replotted from Figure 7c. Agreement is reasonable.

We are finally in a position to say something about the upslope energy variations illustrated in Figure $8 \mathrm{a}$ and the velocity polarization of Figure 9a. Using the odd mode form, Eq. (30) gives to $0\left(l^{1 / 2}\right)$

and

$$
\begin{aligned}
v & =e^{i(k x-\omega t)}\left\{i k \sin l y \frac{\cosh B \kappa z}{\cosh B \kappa}\right. \\
& \left.+\left(\frac{l \epsilon}{\alpha}\right) \frac{\cos l y}{\left(1-\sigma^{2}\right)}(\cos x+i \sigma \sin x) \frac{\cosh B z}{\cosh B}\right\}
\end{aligned}
$$

$$
u=-e^{i(k x-\omega t)}\left\{l \cos l y \frac{\cosh B \kappa z}{\cosh B \kappa}\right\}
$$

The one-dimensional corrugations affect only the north-south velocity component in a region of height $B^{-1} H(\sim 400 \mathrm{~m})$ near the bottom. At the bottom the $v$ component has the form

$$
v=e^{i(k x-\omega t)}\left\{i k \sin l y+\left(\frac{l \epsilon}{\alpha}\right) \frac{\cos l y}{1-\sigma^{2}}(\cos x-i \sigma \sin x)\right\} .
$$

Taking the ratio with the $u$-component gives, in the limit $\sigma<<1$ : 


$$
\frac{v}{u}=-\frac{\epsilon}{\alpha} \cos x+i \sigma\left\{\left(-\frac{\epsilon}{\alpha}\right) \sin x+a \tan l y\right\}
$$

where $a=\tanh B \kappa\left[1+\left(\frac{\epsilon}{\alpha}\right)^{2} \frac{1}{2 \tanh B \kappa}\right] \cong \frac{1}{2}\left(\frac{\epsilon}{\alpha}\right)^{2} \quad$ if $\tanh$ $B \kappa \cong 1$ and $\epsilon / \alpha>>1$. Equation (33) predicts, as $\sigma \rightarrow 0$, an increase in $|v|$ from mooring 572 on the south where $\cos x \cong 0$ to 571 further north where $\cos$ $x \cong 1$ and Eq. (32b) suggests that $|u|$ also increases if $l y<\pi / 2$ as seems likely for the lowest mode. Both predictions are opposite to the observed variations but are also very sensitive to position relative to the corrugations. In the discussion of the mean flow it was found that the local isobath separation was crucial: unfortunately, the linear wave problem with two-dimensional roughness does not appear to be analytically tractable.

With Eq. (34) we can look at the frequency dependence of the velocity polarization. The first term is the local isobath slope and shows that the low frequency motions must tend towards linear polarization (at the bottom) with decreasing frequency. Mooring 572 is just west of a peak in the corrugations so that the first term in (34) is small but positive. As $\sigma$ increases from 0 the second term quickly becomes equal to the first, changing the phase from 0 to $\pm 90^{\circ}$. If the $y$ dependent part of the imaginary term dominates then the phase will tend in the manner observed in Figure 9a. Mooring 571 is near a node in the corrugations such that $\cos x \cong+1$. $u$ and $v$ will be out of phase as $\sigma \rightarrow 0$. However, because the real part of the R.H.S. of (34) is relatively large compared to the imaginary for $\sigma<<1 u$ and $v$ will maintain their $180^{\circ}$ phase relationship to much higher frequencies as is observed in Figure 9a. Aiding this tendency is the additional fact that $\sin x \cong 0$ and the $\tan l y$ term will decrease with frequency increasing.

\section{Summary and conclusions}

Moored observations of current, nine months in duration, at two sites spaced $10 \mathrm{~km}$ apart on the north wall of Charlie Gibbs Fracture Zone show large horizontal and vertical changes in the variance of the mean and low frequency fluctuations. We have attempted to rationalize these changes with quasigeostrophic potential vorticity dynamics and various simplified models of the topography.

Detailed bathymetry in the vicinity of the moorings shows that superimposed on the general slope of the north wall are large amplitude corrugations trending approximately to the north with peak-to-peak amplitude of $600 \mathrm{~m}$ and east-west wavelength $18 \mathrm{~km}$. Vertical velocities determined from conservation of heat are sufficiently small that the mean flow must closely follow isobaths and would be distorted in the observed manner if the isobaths at mooring 572 are further apart than those at 571 .

The time dependent motions are also strongly spatially varying and we have used 
empirical orthogonal functions (Figs. 4-7) to explore the changes in vertical structure with frequency. There are three different frequency bands within which the nature of the motion changes only gradually. At the very lowest (periods 19-96 days) the dominant mode is weakly bottom intensified and mostly east-west. A second mode is also distinguishable: it decreases toward the bottom and both components are approximately equal in amplitude but differ by $90^{\circ}$ in phase (north leading east). At intermediate frequencies (periods less than 19 days but greater than 3.6 days) there is just one significant mode which is bottom trapped with the trapping increasing with frequency and motions changing from being dominantly eastwest to equipartition with frequency (Fig. 7c). At $\omega=.28$ c.p.d. (3.6 days) a transition occurs and two modes again are significant: Now, however, the first $(\sim 50 \%$ of the variance) is highly bottom trapped (decay scale $>400 \mathrm{~m}$ ) and the second ( $\sim 25 \%$ of the variance) more weakly bottom trapped much like the intermediate frequencies.

Inspection of the spectra from the two moorings on the north wall reveals striking changes over the $10 \mathrm{~km}$ north-south separation (Figs. 8 and 9). Firstly, the energy in low frequencies (periods greater than 10 days) decreased by over an order of magnitude moving north at $3000 \mathrm{~m}$ and a somewhat reduced amount at $1000 \mathrm{~m}$. Secondly the near bottom velocity vectors are polarized in such a way as to: (1) be out of phase throughout the subinertial band at a location where the local isobaths are straight and trend to the northwest, and (2) change gradually from being in phase to quadrature at a location where the isobaths are highly curved and near a maximum southward excursion.

By computing the ratio of strength of the nonlinear terms to the linear ones in both the momentum and the heat balances we have shown (Fig. 10) that the timedependent motions under consideration are approximately linear except in the very lowest band $(\omega<.08$ c.p.d.).

We have computed orthogonal dynamical modes of low frequency, quasigeostrophic waves for idealized models of the north wall of CGFZ which attempt to include important features of the topography. Over a plane slope of infinite extent the barotropic mode is a bottom trapped topographic wave, while the baroclinic modes are baroclinic Rossby waves which adjust so as to place a node at the bottom when the slope is much greater than $\beta$. These baroclinic waves, however, can only exist at frequencies lower than those resolved by our analysis and plane waves cannot explain the large horizontal energy gradients. Taking account of the finite extent of the north wall of the Fracture Zone gives more satisfactory results. In the frequency range of interest topographic waves exist over the sloping portion while the baroclinic modes are now evanescent and match incoming energy to reflected and transmitted. Short incoming Rossby waves generate relatively large amplitude baroclinic fringe modes with nodes at the bottom. Long waves can match more easily to weakly bottom trapped topographic waves and have less need for the 
fringe modes which would more closely resemble flat bottom baroclinic modes. An upslope modal structure in the topographic wave component is established. We suggest that the changes in energy level at $1000 \mathrm{~m}$ are a result of the exponential decay in amplitude of the baroclinic fringe modes as well as the changes associated with the horizontal structure of the topographic wave component.

Moderately trapped topographic waves have a maximum frequency given by $N \alpha^{*}$, the Brunt-Väisälä frequency times the bottom slope and this is attained when the wavenumber vector is along the slope. Both plane transverse waves and even cross slope modes predict a ratio of velocity components $|v| /|u|$ which changes from zero at low frequencies to $\infty$ as $\omega \rightarrow N \alpha$, while for the odd modes the ratio remain finite, consistent with our observation of equipartition at higher frequencies. Also favoring the odd mode interpretation is the observed $90^{\circ}$ phase relation between $u$ and $v$ (Fig. 6). A fit of predicted $|v| /|u|$ with observed (Fig. 7c) is rather imprecise but is consistent with 572 , being $9 \mathrm{~km}$ south of the center line of the slope and the maximum frequency $N \alpha^{*} \cong .28$ c.p.d. This gives $\alpha^{*} \cong .023$ a net change of $250 \mathrm{~m}$ over $10 \mathrm{~km}$.

Bottom trapped motions are also observed above this frequency which is, in fact, the point at which two modes appear, the principal one being very highly bottom trapped. In order to interpret this band we have had to go one step further in our modeling of the bathymetry. By modeling the small-scale roughness as north-south sinusoidal corrugations we find that this permits the existence of topographic waves at higher frequencies. The frequency $N \alpha^{*}$ is now identified as a critical point below which there exists a topographic wave somewhat modified by the roughness and above which two waves exist. One travels westward like a super-frequency topographic wave and the other moves eastward and is dominated by a corrugation scale, highly bottom intensified disturbance. In fact, our perturbation scheme breaks down near $\omega^{*}=N \alpha^{*}$ but the form of the result agrees well with the observations.

The effect of the corrugation also helps rationalize near bottom spatial variations in the energy distribution; at low frequencies these variations should be similar to those seen in the mean flow. We have also demonstrated that velocity polarization depends strongly on topography; in regions of straight isobaths (a node in the corrugations) the near bottom velocity remain approximately linearly polarized up to about the maximum allowed frequency, while in regions of curved isobaths (a peak or trough) the velocity components change gradually with frequency from being in or out of phase to quadrature.

Acknowledgments. This is contribution 4343 from the Woods Hole Oceanographic Institution, an investigation supported by the Office of Naval Research under Contract N00014-76-C0197, NR 083-400. We gratefully acknowledge the support of the Buoy Group at the Woods Hole Oceanographic Institution in collecting and processing the data. We are also grateful to Sandy Shor and Roger Searle for providing their version of the CGFZ bathymetry and to the two referees whose suggestions greatly improved the quality of this presentation. 


\section{Appendix: Topographic Waves on a Corrugated Slope}

We wish to solve Eqs. (27a-c) subject to the conditions that $l<<1$ and $l \epsilon / \alpha \sim 0\left(l^{1 / 2}\right)$. We define a two-length scale perturbation procedure according to

and

$$
\frac{\partial}{\partial x}=\frac{\partial}{\partial x^{\prime}}+l \frac{\partial}{\partial x}
$$

$$
\frac{\partial}{\partial z}=B\left[\frac{\partial}{\partial z^{\prime}}+l \frac{\partial}{\partial Z}\right] \text {. }
$$

and expand pressure and $\sigma$ according to

$$
\begin{aligned}
P(x, z)= & P^{(0)}\left(x^{\prime}, z^{\prime}, X, Z\right)+l^{1 / 2} P^{(1 / 2)}\left(x^{\prime}, z^{\prime}, X, Z\right) \\
& +l P^{(1)}\left(x^{\prime}, z^{\prime}, X, Z\right)+\ldots \\
\sigma= & \sigma^{(0)}+l^{1 / 2} \sigma^{(1 / 2)}+l \sigma^{(1)}+\ldots
\end{aligned}
$$

At 0(1):

$$
P_{x^{\prime} z^{\prime}}{ }^{(0)}+P_{x^{\prime} z^{\prime}}{ }^{(0)}=0
$$

with

$$
P_{z^{\prime}}{ }^{(0)}=0 \text { on } z^{\prime}=0
$$

and

$$
i \sigma^{(0)} P_{z^{\prime}}{ }^{(0)}=P_{z^{\prime}}{ }^{(0)} \text { on } z^{\prime}=-B .
$$

This set of equations has only the solution that

$$
P^{(0)}\left(x^{\prime}, z^{\prime}, X, Z\right)=P_{0}(X, Z)
$$

At lowest order the pressure amplitude is unaffected by the corrugations. At $0\left(l^{1 / 2}\right)$ :

with

$$
P_{z^{\prime} g^{\prime}}^{(1 / 2)}+P_{z^{\prime} z^{\prime}}^{(1 / 2)}=0
$$

and

$$
P_{z^{\prime}}^{(1 / 2)}=0 \text { on } z^{\prime}=0
$$

$$
\begin{aligned}
& i \sigma^{(0)} P_{z^{\prime}}^{(1 / 2)}=P_{z^{\prime}}^{(1 / 2)}-i\left(\frac{\epsilon}{\alpha} l^{1 / 2}\right) \cos x^{\prime} P_{0}(X, Z) \\
& \text { on } z^{\prime}=-B \\
& \text { or } Z=-B l \text {. }
\end{aligned}
$$

The solution is

$$
P^{(1 / 2)}\left(x^{\prime}, z^{\prime}, X, Z\right)=\left(\frac{\epsilon}{\alpha}\right) l^{1 / 2} \frac{1}{\left(1-\sigma^{(0) 2}\right)} P_{0}(X,-B l)\left[-\sigma^{(0)} \cos x^{\prime}+i \sin x^{\prime}\right] \frac{\cosh B z^{\prime}}{\cosh B}
$$
and we have taken $\tanh B=1$.

At $0(l)$ :

with

$$
P_{z^{\prime} z^{\prime}}^{(1)}+P_{z^{\prime} z^{\prime}}^{(1)}=0
$$

and

$$
\frac{\partial}{\partial z^{\prime}} P^{(1)}+\frac{\partial}{\partial Z} P^{(0)}=0 \text { on } z^{\prime}=Z=0
$$

$$
\begin{aligned}
i \sigma^{(0)} P_{z}^{(0)}+i \sigma^{(1 / 2)} P_{z^{\prime}}^{(1 / 2)}=P_{X}{ }^{(0)}+P_{x^{\prime}}{ }^{(1)}- & i\left(\frac{l^{1 / 2} \epsilon}{\alpha}\right) \cos x^{\prime} P^{(1 / 2)} \\
& \text { on } z^{\prime}=-B \\
& \text { or } Z=-B l .
\end{aligned}
$$

The terms depending on the large scales, $X$ and $Z$ alone, will lead to secular growth unless they cancel. Therefore 
and

$$
P_{\mathrm{oz}}=0 \text { on } Z=0
$$

$$
i \sigma^{(0)} P_{0 z}=P_{0 X}+\frac{i}{2}\left(\frac{l^{1 / 2} \epsilon}{\alpha}\right)^{2}\left(\frac{\sigma^{(0)}}{1-\sigma^{(0) 2}}\right) P_{0} \quad \text { on } \quad Z=-B l
$$

which are boundary conditions for the lowest order pressure field. Canceling singularities in the governing equations at $0\left(l^{2}\right)$ gives:

$$
P_{0 x x}+P_{0 z z}-P_{0}=0 .
$$

The solution satisfying (A.7a) and (A.7b) is

with $\left(\sigma \equiv \sigma^{(0)}\right)$

$$
P_{0}=e^{i(k x / l)} \frac{\cosh (K Z / l)}{\cosh K B}, \quad K^{2}=k^{2}+l^{2},
$$

$$
\sigma \tanh B K=-\frac{k}{K}-\frac{1}{2} l^{2}\left(\frac{\epsilon}{\alpha}\right)^{2} \frac{\sigma}{1-\sigma^{2}}
$$

To $0\left(l^{1 / 2}\right)$ in the original scaled coordinates, $x$ and $z$ :

$$
P(x, z)=e^{i k x} \frac{\cosh K B z}{\cosh K B}+l \frac{\epsilon}{\alpha} \frac{1}{1-\sigma^{2}}(-\sigma \cos x+i \sin x) \frac{\cosh B z}{\cosh B}+\ldots
$$

These are the forms used for pressure and the dispersion relation to the text.

It is of interest to calculate two limits. As $\sigma \rightarrow 0$ (A.9) gives

$$
\sigma\left[\tanh B K+\frac{1}{2}\left(\frac{l \epsilon}{\alpha}\right)^{2}\right]=-\frac{k}{K}
$$

so that, for a given orientation of the wavenumber vector the frequency is reduced. Equation (A.10) gives as $\sigma \rightarrow 0$

$$
P(x, z) \rightarrow e^{\imath k x} \frac{\cosh B K z}{\cosh B K}-l \frac{\epsilon}{\alpha}(i \sin x-\sigma \cos x) \frac{\cosh B z}{\cosh B}
$$

which assures that the velocity vector is along the isobaths at the bottom when $\sigma=0$.

On the other hand, as $\sigma \rightarrow 1$ there are two possibilities corresponding to the westward and eastward propagating waves. For westward waves $l \rightarrow 0$ and $k \rightarrow K$ as $\sigma \rightarrow 1$ while $l \rightarrow 0$ and $k \rightarrow-K$ as $\sigma \rightarrow 1$ for eastward waves. In order to determine the effect on the pressure we must determine the form of $(l \epsilon / \alpha) /\left(1-\sigma^{2}\right)$ in (A.10). It can be shown that $\left(\frac{l \epsilon}{\alpha} \frac{1}{\sigma^{2}-1}\right) \rightarrow$ $|K|^{1 / 2}$ when $k \rightarrow-K$ but $\left(\frac{l \epsilon}{\alpha}\right) \frac{1}{\sigma^{2}-1} \rightarrow\left(2 \frac{K}{\sigma-1}\right)^{1 / 2}$ when $k \rightarrow K$ and the perturbation scheme breaks down near $\sigma=1$ for the eastward propagating waves.

\section{REFERENCES}

Bryden, H. L. 1976. Horizontal advection of temperature for low-frequency motion. Deep-Sea Res., 23, 1165-1174.

Lonsdale, P., and A. Shor. 1979. The oblique intersection of the Mid-Atlantic Ridge with Charlie-Gibbs transform fault. Technophysics, in press.

McWilliams, J. C. 1974. Forced transient flow and small scale topography. Geophys. Fl. Dyn., 6, 49-79.

McWilliams, J. C., and G. Flierl. 1976. Optimal, quasigeostrophic wave analysis of MODE array data. Deep-Sea Res., 23, 285-300.

Rhines, P. B. 1969. Slow oscillations in an ocean of varying depth-I Abrupt topography. J. Fluid Mech., 37, 161-189. 
1970. Edge-, bottom- and Rossby waves in a rotating stratified fluid. Geophys. Fl. Dyn., 1, 273-302.

Rhines, P. B., and F. Bretherton. 1973. Topographic Rossby waves in a rough-bottomed ocean. J. Fluid Mech., 61, 583-607.

Richman, J. G., C. Wunsch, and N. G. Hogg. 1977. Space and time scales of mesoscale motion in the western North Atlantic. Rev. Geophys. and Sp. Phys., 15, 385-420.

Schmitz, W. J., Jr. 1974. Observations of low-frequency current fluctuations on the Continental Slope and Rise near Site D. J. Mar. Res., 32, 233-251.

Schmitz, W. J., Jr., and N. G. Hogg. 1978. Observations of energetic low frequency fluctuations in the Charlie-Gibbs Fracture Zone. J. Mar. Res., 36, 725-734.

Shor, A., P. Lonsdale, C. Hollister, and D. Spencer. 1980. Charlie-Gibbs Fracture Zone: Deep and bottom water transport (in preparation).

Suarez, A. 1971. The propagation and generation of topographic oscillations in the ocean. Ph.D. Thesis, Deptment of Meteorology, M.I.T.

Thompson, R. 1977. Observations of Rossby waves near Site D. Prog. in Oceanogr., 7, 135-162. Thompson, R., and J. R. Luyten. 1976. Evidence for bottom trapped topographic Rossby waves from single moorings. Deep-Sea Res., 23, 629-635.

Wallace, J. M., and R. F. Dickinson. 1972. Empirical orthogonal representation of time series in the frequency domain. Part I: Theoretical considerations. J. Appl. Meteor., 11, 887-892.

Received: 22 March, 1979; revised: 11 February, 1980.

Printed in U.S.A. for the Sears Foundation for Marine Research, Yale University, New Haven, Connecticut, 06520, U.S.A. Van Dyck Printing Company. North Haven, Connecticut, 06473, U.S.A. 


\section{MANDATORY DISTRIBUTION LIST}

FOR UNCLASSIFIED TECHNICAL REPORTS, REPRINTS, AND FINAL REPORTS

PUBLISHED BY OCEANOGRAPHIC CONTRACTORS

OF THE OCEAN SCIENCE AND TECHNOLOGY DIVISION

OF THE OFFICE OF NAVAL RESEARCH

(REVISED NOVEMBER 1978)

1 Deputy Under Secretary of Defense

(Research and Advanced Technology)

Military Assistant for Environmental Science

Room 30129

Washington, D.C. 20301

Office of Naval Research

800 North Quincy Street

Arlington, VA 22217

3 ATTN: Code 483

1 ATTN: Code 460

2 ATTN: 102B

1 CDR J. C. Harlett, (USN)

ONR Representative

Woods Hole Oceanographic Inst.

Woods Hole, MA 02543

Commanding Officer

Naval Research Laboratory

Washington, D.C. 20375

6 ATTN: Library, Code 2627
12 Defense Documentation Center Cameron Station

Alexandria, VA 22314

ATTN: DCA

Commander

Naval Oceanographic Office

NSTL Station

Bay St. Louis, MS 39522

ATTN: Code 8100

ATTN: Code 6000

ATTN: Code 3300

$1 \quad$ NODC/NOAA

Code D781

Wiscons in Avenue, N.W.

Washington, D.C. 20235 
UNCLASSIFIED $4 / 81$

SECURITY CLASSIFICATION OF THIS PAGE (When Data Entored)

\begin{tabular}{|c|c|}
\hline REPORT DOCUMENTATION PAGE & $\begin{array}{l}\text { READ INSTRUCTIONS } \\
\text { BEFORE COMPLETING FORM }\end{array}$ \\
\hline \begin{tabular}{l|l} 
1. REPORT NUMBER & 2. GOVT ACCESSION NO. \\
WHOI $-81-23$ &
\end{tabular} & 3. RECIPIENT'S CATALOG NUMBER \\
\hline \multirow{2}{*}{$\begin{array}{l}\text { 4. TITLE (and Subtitlo) } \\
\text { A DYNAMICAL INTERP?ETATION OF LOW FREQUENCY } \\
\text { MOTIONS NEAR VERY ROUGH TOPOGRAPHY -THE CHARLIE } \\
\text { GIBBS FRACTURE ZONE }\end{array}$} & $\begin{array}{l}\text { 5. TYPE OF REPORT A PERIOD COVERED } \\
\text { Technical }\end{array}$ \\
\hline & $\begin{array}{l}\text { 6. PERFORMING ORG. REPORT NUMBER } \\
\text { WHOI Cont. \# } 4343\end{array}$ \\
\hline $\begin{array}{l}\text { 7. AUTHOR(a) } \\
\text { N.G.Hogg and W.J.Schmitz, Jr. }\end{array}$ & $\begin{array}{l}\text { 8. CONTRACT OR GRANT NUMBER(a) } \\
\text { N00014-76-C-0197; }\end{array}$ \\
\hline $\begin{array}{l}\text { 9. PERFORMING ORGANIZATION NAME AND ADDRESS } \\
\text { Woods Hole Oceanographic Institution } \\
\text { Woods Hole, Massachusetts, } 02543\end{array}$ & $\begin{array}{l}\text { 10. PROGRAM ELEMENT, PROJE CT, TASK } \\
\text { AREA Q WORK UNIT NUMBERS } \\
\text { NR } 083-400\end{array}$ \\
\hline \multirow{2}{*}{$\begin{array}{l}\text { 11. CONTROLLING OFFICE NAME AND ADDRESS } \\
\text { NORDA } \\
\text { National Space Technology Laboratory } \\
\text { Bay St. Louis. MS } 39529\end{array}$} & $\begin{array}{l}\text { 12. REPORT DATE } \\
\text { Apri } 11981\end{array}$ \\
\hline & 13. NUMBER OF PAGES \\
\hline \multirow[t]{2}{*}{ 14. MONIT JRING AGENCY NAME \& ADDRESS(It different from Controllind Oflice) } & $\begin{array}{l}\text { 15. SECURITY CLASS. (of thie roport) } \\
\text { Unclassified }\end{array}$ \\
\hline & $\begin{array}{l}\text { 15a. DECLASSIFICATION/DOWNGRADING } \\
\text { SCHEDULE }\end{array}$ \\
\hline
\end{tabular}

16. DISTRIBUTION STATEMENT (of this Roport)

Approved for public release; distribution unlimited.

17. DISTRIBUTION STATEMENT (of the abotract ontered in Block 20, il difforent (rom Roport)

18. SUPPL EMENTARY NOTES

Reprinted from: Journal of Marine Research 38 (2): 215-248 (1980).

19. KEY WORDS (Continue on reverse a to if noceseary and identify by block number)

1. Low frequency motions

2. Topographics waves

3. Gibbs Fracture Zone

20. ABSTRACT (Continue on roverae ai of neceecary and identlfy by block numbse)

See reverse side. 
As a sequel to Schmitz and Hogg (1978), nine-month moored observations of current and temperature from the Charlie Gibbs Fracture Zone are further described, and then interpreted in terms of low frequency quasigeostrophic motions. Large vertical and horizontal changes are observed in the variance of both mean and fluctuating components. It is demonstrated that these changes could be associated with the (complex) nature of the topography.

With regard to the mean flow, it is shown through an advective model that the topography is sufficiently steep to force this motion to closely follow isobaths. Time-dependent motions for periods from 2 to 96 days are described using the technique of empirical orthogonal functions. The most energetic mode is always bottom trapped, with flow oriented along isobaths at lower frequencies and approaching equipartition of along-and cross-isobath motions at higher frequencies. At the lowest frequencies a second mode which increases upward in energy is also judged significant, while for periods shorter than 3.6 days the second mode is again highly bottom trapped. We interpret these motions using linear wave theory. There is relatively close correspondence between theory and observation when the effects of both largeand small-scale topographic features are included in the model calculations. In addition to the usual topographic wave, the abrupt slope changes on the north wall allow for a baroclinic fringe mode with a near bottom node at low frequnecies and small-scale bottom corrugations force highly bottom trapped waves above the smooth slope cut-off frequency. 

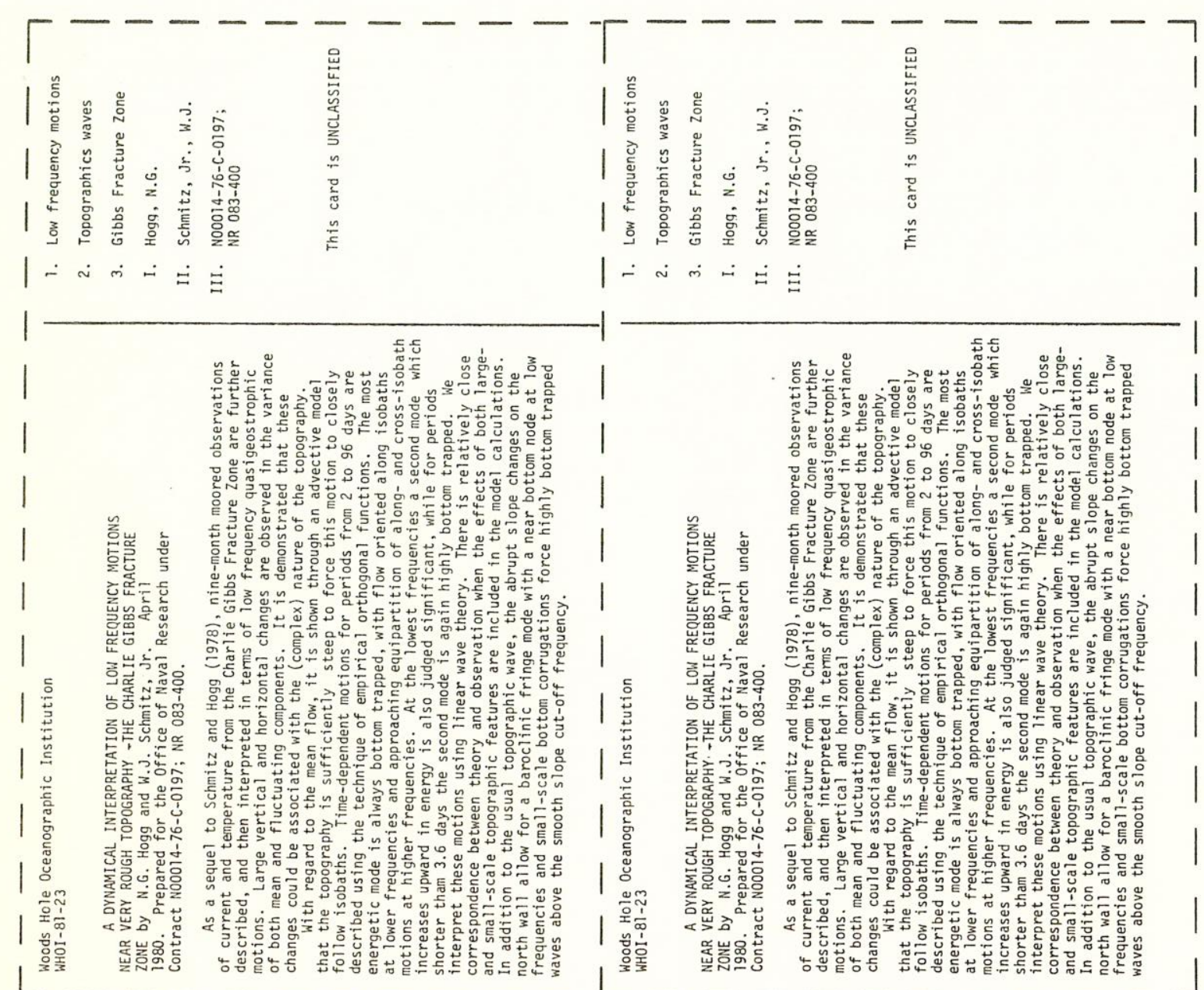

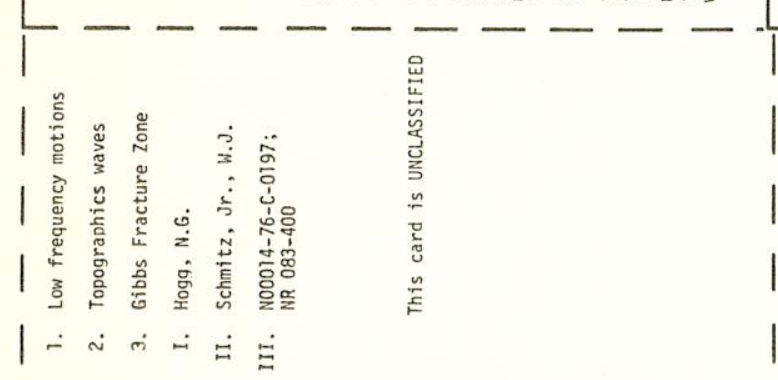

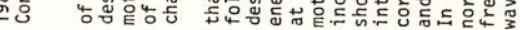
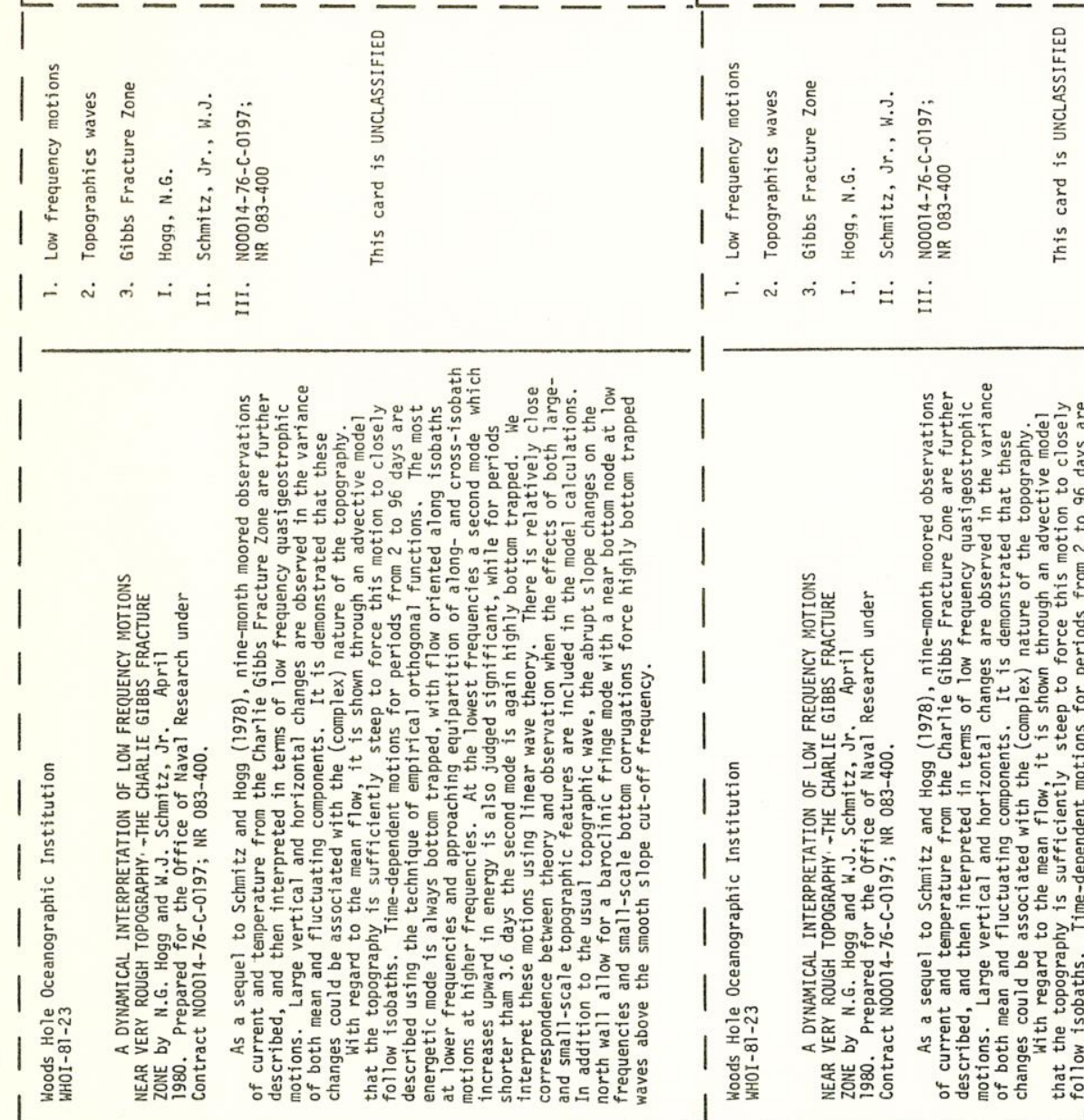

- 